\title{
Nanomaterials, Soft Matter, and Electronic Materials Find Common Ground at 2003 MRS Spring Meeting
}

The interplay of materials with biology took center stage at the 2003 Materials Research Society Spring Meeting, which had both the meeting's plenary speaker, David A. Tirrell of the California Institute of Technology, and the MRS Outstanding Young Investigator, Tim Deming of the University of California-Santa Barbara, presenting talks with strong biological content. This topic was further explored in Symposium O on Materials Inspired by Biology and in one of the presentations in Symposium X on Frontiers of Materials Research. Also, one of the four clusters of symposia covered Molecular Materials and Biomaterials.

Held April 21-25 at the San Francisco Marriott and Argent Hotels in San Francisco, California, the Meeting presented symposia clustered under Electronic and Optical Materials, Nanostructured Materials, and General, in addition to the bio-related cluster.

The Meeting-chaired by Terry Garino (Sandia National Laboratories), Hans-Joachim Gossmann (Axcelis Technologies), Lisa Klein (Rutgers University), and Albert Polman (FOM-Institute AMOLF) incorporated tutorial sessions on exciting areas of research; a series of lunchtime talks on Frontiers of Materials Research for a nonspecialist audience; an afternoon session on "Materials Research to Meet 21st Century Defense," by the U.S. National Materials Advisory Board along with evening seminars by various funding agencies; and other special events. The Meeting reached over 2600 attendees.
The 25 technical symposia were categorized into clusters, with about 1300 oral presentations and nearly 900 posters. The equipment exhibit featured over 90 exhibitors from around the world, displaying a range of equipment, services, products, publications, and software. Once again MRS hosted a Career Center providing valuable services for meeting attendees, including access to current job postings, a resume file for prospective employers to review, and on-site interview opportunities. Graduate Student Gold and Silver Awards were announced as well as awards for best poster presentations. See sidebars covering these and other events. Also see daily meeting news and highlights on the MRS Web site for more detailed reports of the technical content (www.mrs.org/meetings).

\section{Technical Talks \\ Biomaterials}

"Beautiful work, as usual," said one participant about M. Mrksich's (Univ.
Chicago) presentation on "Tailored Interfaces to Integrate Cells with Electronics" in Symposium N. Such integration has significant potential because cells are functional and able to produce power, sense, and synthesis abilities that are not yet easily engineered. But integrating them with inorganic materials can be a challenge. As an example, Mrksich focused on the "sensing" ability of materials, noting how cells communicate through molecular recognition, while communication in electronics is through current and voltage. First, the two materials need to adhere to each other. The strategy Mrksich described is based on self-assembly of monolayers of alkanethiolates on gold that are then modified with peptide ligands to promote cell adhesion. The cells recognize the peptides, attach, and spread. The monolayers are engineered with electroactive moieties so an electrical potential applied to the gold film results in a change in the activities of ligands.

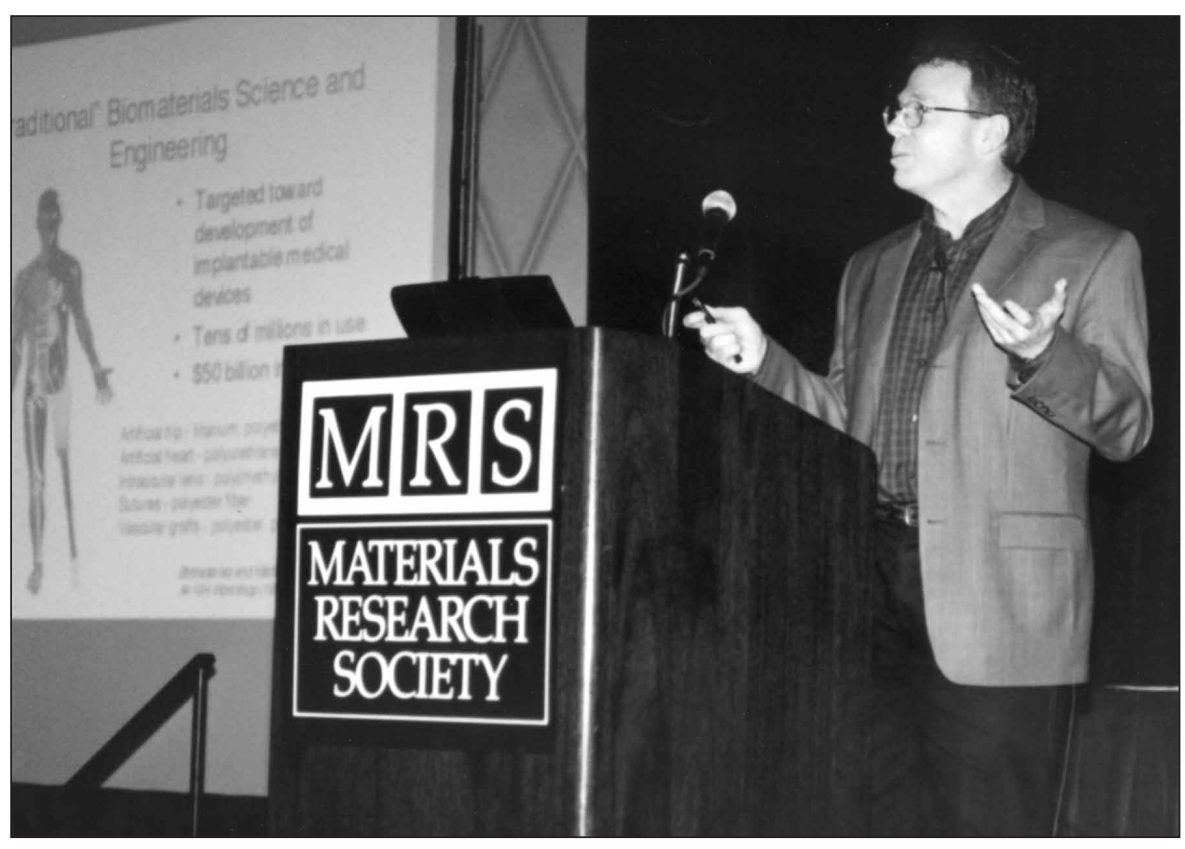

Plenary speaker David A. Tirrell of California Institute of Technology presented his group's research on genetically directed synthesis of novel macromolecules, focusing on a protein engineering approach to vascular reconstruction and regeneration. He said the vascular wall can be examined as a materials system, which needs to have a Young's modulus in the 0.3-0.6 MPa range and also needs to allow for growth of endothelial cells. Tirrell also detailed expression of genetic information on multiple length scales and control of molecular architecture through expression of artificial genes. 
Mrksich also presented initial progress toward "listening" to cells to translate cell signals into electrical ones.

One of the tutorials held at the Spring Meeting was on tissue engineering, as part of Symposium O. This tutorial was designed to introduce materials researchers to the important roles that materials play in the engineering of new tissues and organs. In an introduction to and overview of soft-tissue engineering, A. Panitch (ASU) said that two corresponding issues need to be considered: how the body responds to a material at the molecular and cellular levels, and how a material responds to the body including effects such as surface modification, erosion, degradation, and chemical and/or mechanical failure. Panitch discussed various engineering aspects involving the placement of a foreign body in a biological environment, and how a material can be designed for tissue engineering. She described the use of degradable materials and hydrogels. She also gave various examples of materials used in nerve regeneration.

W. Landis (Northeastern Ohio Univ.) followed with a discussion on hard tissues, giving an exposition on tissue engineering of mineralizing connective tissues. Landis covered vertebrate calcifying tissues and cells, methods of cell culture, choice of scaffolds for tissue engineering, and cell/scaffold construction design. As an example of an engineering calcified model, he described tissue engineering of the small joints in the human hand, which includes fabrication of biodegradable polymers in the shape of the phalangeal bones of the human hand and engineering the small joint using combinations of osteoblasts, chondrocytes, tenocytes, and biodegradable polymers. Landis concluded with a discussion of analytical approaches to documenting viable constructs. D. Mooney (Univ. Michigan) discussed recent research on tissue engineering and regeneration.

\section{Nanostructured Materials}

$\mathrm{V}$. Colvin (Rice) gave an inspiring talk within Symposium Q, Unconventional Approaches to Nanostructures, on the use of protein crystals as templates for organizing 3D nanoscale materials. The use of protein crystals allows for the creation of structures that are not limited by the so-called "sphere theory" as is the case when using inorganic colloidal templates. Colvin showed results achieved with lysozyme and crystals of the cowpea mosaic virus (CPMV). The key issue for obtaining a robust and macroscopic crystal for processing is the use of crosslinking stabilization. In order to fill in the large pore volume of the crystals, Colvin showed that they could be infiltrated by a variety of schemes such as electroless plating in the case of metals, and by polymer or ceramic fillings. Among other results, Colvin showed images of Pt-filled CPMV crystals. The synthesized materials have potential uses in catalytic, electrical, and optical applications.

Symposium S on Nanoscale Thermal Transport included a talk by K. Goodson (Stanford) on electrothermal phenomenon in nanotransistors. The reliability of future nanoscale transistors will depend on efficient dissipation of generated heat. It is important, therefore, to understand nanoscale heat generation in semiconductors. Goodson described recent modeling and experimental results using coupled electrothermal Monte Carlo simulations of nanotransistors and measurements of hotspot formation in semiconductors. Also, current new geometries such as doublegate SOI, FinFET, and PillarFET will be affected by heat generation and Goodson discussed results for these as well. Goodson also summarized recent results on measuring subcontinuum thermal resistance near a hotspot in $\mathrm{Si}$.

\section{ACRONYM KEY}

2D: two-dimensional

3D: three-dimensional

AFB: Air Force Base

AFRL: U.S. Air Force Research Laboratory

AIST: Advanced Industrial Science and Technology

ANL: Argonne National Laboratory

ANU: Australian National University

ARO: Army Research Office

ASU: Arizona State University

BioMEMS: biomicroelectromechanical systems

BP: British Petroleum

Caltech: California Institute of Technology

CEA: Centre Energie Atomique

CMOS: complementary metal oxide semiconductor

CMP: chemical-mechanical polishing

CNM: Centro Nacional de Microelectrónica, Barcelona

CNRS-ONERA: Centre National de la Recherche Scientifique-Office National d'Etudes et de Recherches Aerospatiales

CNT: carbon nanotube

CSIC: Consejo Superior de Investigaciones Científicas

CVD: chemical vapor deposition

DARPA: Defense Advanced Research

Projects Agency

ETH: Ergonische Technische Hochschule
EXAFS: extended x-ray absorption

fine structure

FET: field-effect transistor

FIB: focused ion beam

GE: General Electric

IIT: Indian Institute of Technology

INFM: L'Istituto Nazionale per la Fisica della

Materia, Italy

KTH: Royal Institute of Technology

LANL: Los Alamos National Laboratory

LED: light-emitting diode

LEM: Laboratoire d'Etudes des Microstructures

LLNL: Lawrence Livermore National Laboratory

MAPLE: matrix-assisted pulsed laser evaporation

MBE: molecular-beam epitaxy

MEMS: microelectromechanical systems

MFM: magnetic force microscopy

MIT: Massachusetts Institute of Technology

MPI: Max Planck Institute

MTS: methyltrichlorosilane

NASA: National Aeronautics and Space Administration

NCSU: North Carolina State University

NDR: negative differential resistance

NIH: National Institutes of Health

NIST: National Institute of Standards and

Technology

NMAB: National Materials Advisory Board
NREL: National Renewable Energy Laboratory NRL: Naval Research Laboratory

NSF: National Science Foundation OLED: organic light-emitting diode ONR: Office of Naval Research PARC: Palo Alto Research Center PLD: pulsed laser deposition PLY: photoluminescence yield PMMA: poly(methyl methacrylate) PSU: Pennsylvania State University rf: radio frequency

RPI: Rensselaer Polytechnic Institute SNL: Sandia National Laboratories

SOI: silicon-on-insulator

TCO: transparent conducting oxide

TEM: transmission electron microscopy

TEY: total electron yield

TFT: thin-film transistor

TI: Texas Instruments

UC: University of California

UCLA: University of California-Los Angeles UCSB: University of California-Santa Barbara UCSD: University of California-San Diego UT: University of Texas

UV: ultraviolet

WSI: Walter Schottky Institute, Munich XRD: x-ray diffraction 


\section{Electronic and Optical Materials}

L. Rea (AFRL) opened a special session of invited talks in Symposium C on New Applications for Wide-Bandgap Semiconductors, in which she gave an overview and the current status of wide-bandgap semiconductors. Tremendous progress has been seen in the development of devices based on wide-bandgap semiconductor materials. However, this is likely just the tip of a "product iceberg." Rea discussed several anticipated advances in the field such as rf and power devices. She said that a lot can be learned from past developmental efforts such as for GaAs and InP. She reiterated that widebandgap materials technology is not mature enough for it to be declared a success. However, it is likely that success could be dictated by the (commercial) market which could drive prices down.

H. Dietrich (ONR) followed with a discussion of the use of wide-bandgap devices in the future. Clearly $\mathrm{SiC}$ and $\mathrm{GaN}$ devices are of great interest because of the high power densities that can be generated. There are collateral properties of these materials that enhance their utility, including high-voltage operations and higher efficiencies. Dietrich described powerswitching applications of interest to the U.S. Department of Defense, including the all-electric ship, more electric aircraft, and all-electric combat vehicles.

In a provocatively titled presentation "News from the ' $\mathrm{M}$ ' in $\mathrm{CMP}$-Viscosity of CMP Slurries, a Constant?" within Symposium F, W. Lortz (Aerosil \& Silanes, Germany) said that, while the viscosity of CMP slurries is considered to be constant for various calculations, the real viscosity can vary widely at high shear rates. Depending upon the contact region between the polishing pad and the wafer, and how small this distance is based on the asperities on the surface, the shear rates can vary significantly. At low shear rates, surface chemistry is important while at high shear rates, hydrodynamics becomes important. Lortz concluded that viscosity is certainly not constant; however, additional work is required to get more detailed information.

The session on anomalous phenomena in photonics in Symposium J included both theoretical and experimental work on the building of metamaterials with negative refractive index, a topic of great current interest, and of nanostructured metals for the control and enhancement of the transmission through subwavelength apertures. Concerning the latter, F.J. Garcia-Vidal (Universidad Autonoma de Madrid) presented a theoretical study on the properties of surface plasmon

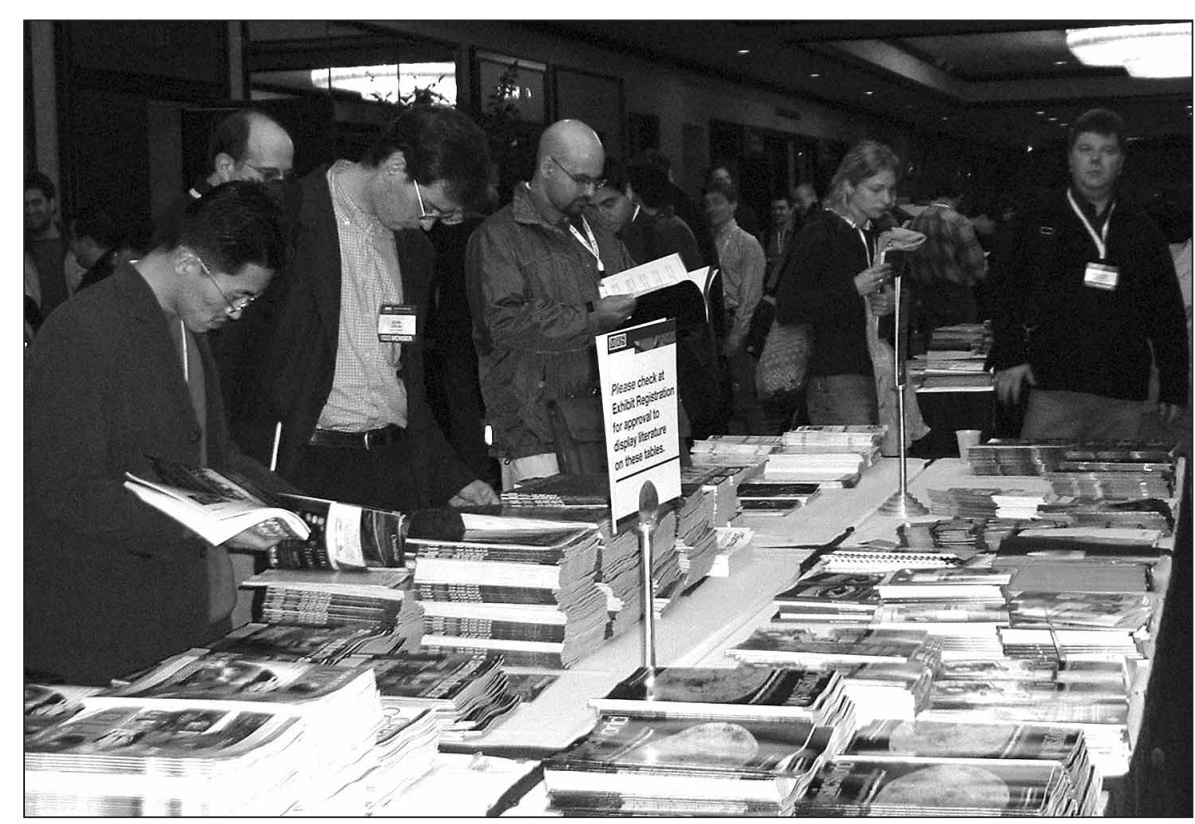

Attendees browse through corporate literature along with journals, conference announcements, and information from other societies, available in the San Francisco Marriott Hotel.

polariton excitation through a single subwavelength aperture in a metal film. He explained how the enhanced transmission can be achieved and, moreover, modulated by creating periodic corrugated structures both at the entry and at the exit sides of the metal film. He demonstrated how beaming and lensing effects can be controlled by choosing suitable geometrical parameters in the periodic structure around the aperture. His results show a surprising effect in which light along an 8-mm structure of 16 grooves can be collected and put through a 40-nm width aperture. Later in the same session, $\mathrm{H}$. Lezec (Univ. Louis Pasteur, Strasbourg) showed experimental results on enhanced transmission through subwavelength patterned hole arrays in $\mathrm{Ag}$ films. He also showed experimental results on the structures earlier discussed by Garcia-Vidal. The beaming effects were clearly demonstrated. Lezec discussed his results in terms of evanescent wave fields.

In another session in Symposium J, P.G. Kik (Caltech) presented his work on subdiffraction focusing and imaging in the near field. Kik presented finite-difference timedomain simulations using the refractiveindex dispersion functions of real materials $\left(\mathrm{Si}_{3} \mathrm{~N}_{4}\right.$ and $\left.\mathrm{Ag}\right)$. His results show how the near-field distribution of an oscillating dipole can be imaged through a $30 \mathrm{~nm}$ $\mathrm{Si}_{3} \mathrm{~N}_{4} / 30 \mathrm{~nm} \mathrm{Ag}$ structure due to the buildup of surface plasmons at the Ag- silicon nitride interface. This special type of focusing could have applications in optical data storage and near-field optical lithography. In another presentation, S. Maier (Caltech) showed both simulations and experimental results for evidence of energy transport in nanoparticle plasmon waveguides. Waveguides of $50 \mathrm{~nm} \mathrm{Au}$ spherical particles prepared by lithography and liftoff clearly showed the energy transport, but they are limited by a high loss of $6 \mathrm{db} / 30 \mathrm{~nm}$. This result can be improved by the generation of structures formed by rods, and indeed Maier showed how in a waveguide system formed of $\mathrm{Ag}$ rods $(90 \times 30 \times 30 \mathrm{~nm})$ the attenuation can be reduced to $6 \mathrm{db} / 200 \mathrm{~nm}$. These results open the road to the creation of optical functional waveguides formed by arrays of metal nanoparticles.

Symposium I on Optoelectronics of Group-IV-Based Materials was preceded with a tutorial in which G. Davies (King's College, London) discussed optical properties of silicon, with an emphasis on luminescence transitions occurring between different electronic states of point defects in silicon. Silicon is by far the most widely used material in electronics. However, because of its indirect gap, $\mathrm{Si}$ is generally considered not suitable for light emitters. Si-based devices would be very useful in implementing optical functions in integrated circuits. Davies addressed three questions: What 
electronic states are available in $\mathrm{Si}$; what properties of the electronic states can be measured; and what obstacles exist for obtaining luminescence from $\mathrm{Si}$.

Next in the tutorial, W. Jantsch (Johannes Kepler Univ., Linz, Austria) elaborated on different approaches to obtaining light from $\mathrm{Si}$, including $\mathrm{Si}-\mathrm{Ge}$, porous Si, Si nanocrystals, and silicides. Jantsch discussed one particular system in detail, Si:Er, which is a rare-earth-doped single-crystal Si. He said that further developments in the foreseeable future include industrial application in simple devices, incorporation into waveguide structures and resonators, and $\mathrm{Er}$ in Sinanocrystals embedded in $\mathrm{SiO}_{2}$. J. Zavada (ARO) gave a brief presentation of recent developments in the field of integration of III-V-based emitting structures with the Si platform.

\section{Other Topics}

The cluster of symposia encompassing general areas included semiconductor spintronics (Symposium V), multiscale phenomena in materials (Symposium W), advanced optical processing of materials (Symposium Y), and electrochemical deposition and corrosion (Symposium Z). As part of Symposium W, V. Bulatov (LLNL) discussed screw dislocations in bcc metals that can move out of plane, and addressed whether screw dislocations in a bcc structure behave canonically. Current understanding is that the dislocation motion mode is delineated by Peierls stress. Below the Peierls stress, dislocation motion occurs by a thermally activated motion by kink-pair mechanisms. Above this threshold, phonon drag controls motion. Bulatov described the results of molecular-dynamics simulations that indicated that dislocation motion in bcc iron occurs by roughening of screw dislocations and the production of a large number of debris loops in the wake of subsequent motion. This occurs without the Peierls threshold transition. Dislocation behavior depends on its length that controls the kinks formed, and the transition from smooth kink mechanisms to rough motion depends on length, temperature, and the stress. Bulatov said that these results could have implications for crystal strength, particularly in the high-strain-rate regime.

D. Norton (Univ. of Florida) opened Symposium Y, Advanced Optical Processing of Materials, with a review of recent advances and challenges of PLD of oxides. He focused on the deposition of oxides for functional electronics using the advantages that PLD offers, especially atomic control during oxide growth and

\section{Marie D'lorio Addresses Attraction and Retention of Women in MSE}

The featured speaker at the Women in Materials Science and Engineering Breakfast was Marie D'Iorio, director of components and technology at the Institute for Microstructural Sciences within the National Research Council of Canada. D'Iorio spoke about the challenging issues associated with attracting and retaining women in science and engineering. Beginning with K-12 education, D'Iorio addressed the importance of using the most contemporary frontiers of human knowledge to capture students' interest, such as the human genome at interfaces between fields such as physics and biology (e.g., growing neurons on imprinted substrates).

D'Iorio said a solid curriculum should be developed to hold that interest throughout the educational cycle. The curriculum should be complemented with small class sizes, strong teachers, and student participation in cooperative education programs and scientific outreach activities. Indeed, D'Iorio cited an American Physical Society (APS) survey of women scientists who reported that their interest in physics started early in high school. D'Iorio emphasized the importance for students to work as part of a science team.

In reiterating the APS survey results, D'Iorio pointed to the majority of respondents who could identify a strong "science mentor," and to a very large percentage of the survey participants who could identify a "positive experience" as an undergraduate student pursuing a science degree. Retention of women in science and engineering, however, raised many issues, such as the need to decrease the length of a postdoctoral assignment thus lowering insecurity and relocation anxiety, the availability of mentors in the workplace, and the chance to "pause the career clock" for family-related issues and support to "re-integrate" after a family-related leave.

While there are still many challenges to attracting and retaining women in science and engineering, D'Iorio said there is hope for the future. More women are being attracted to science and building scientific careers. These successful women scientists are becoming role models and mentors for the next generation. D'Iorio concluded her presentation by challenging the almost 100 people in attendance to get involved in scientific outreach and mentoring.

This event, organized by the MRS Public Outreach Subcommittee, was sponsored by the American Chemical Society, Colloid and Surface Chemistry Division; Aldrich Chemical Company, Inc.; and Gatan, Inc.

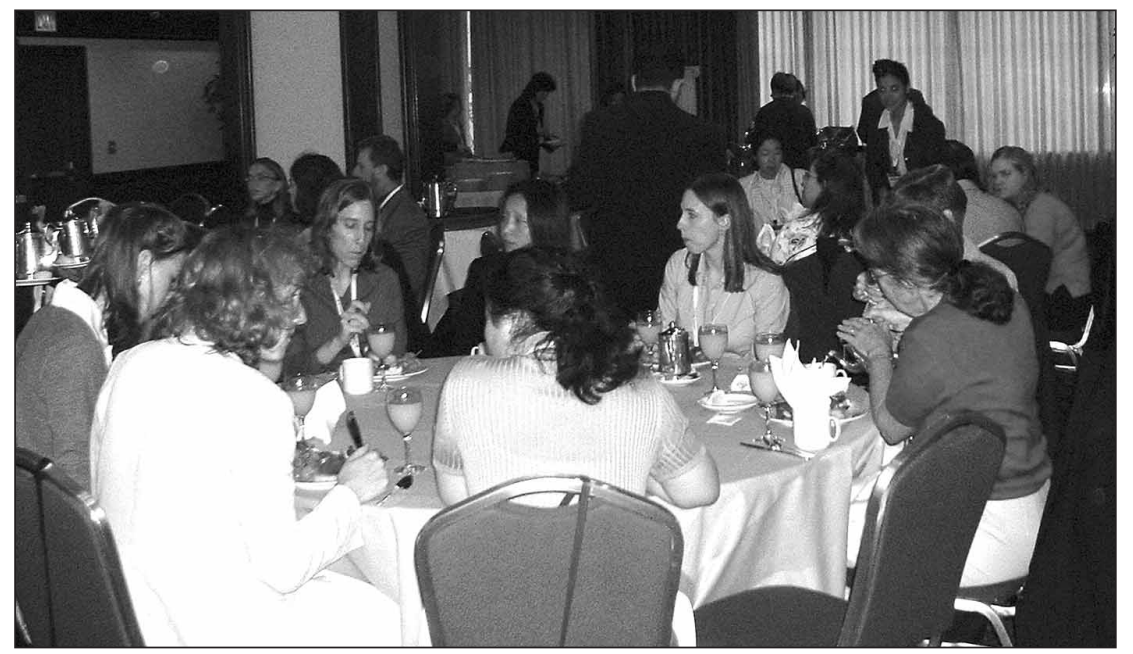


the use of reactive oxidation to control the oxidation states of layers and interfaces. Among other results, Norton showed examples of the deposition of $\mathrm{ZnO}$ for the development of transparent electronics in which the main challenge is the achievement of both $p$ - and $n$-doping in order to build transparent electronic devices. As an example of the control of the oxidation state of layers, Norton showed how the valence of $\mathrm{Ti}$ could be controlled during the epitaxial growth of $\mathrm{LaTiO}_{3}$ by using a hydrogen-reducing atmosphere. He also showed recent results on the preparation of FET devices and UV-emitting diodes based on $\mathrm{ZnO}$ structures. As for future directions, Norton mentioned the improvement of atomic control during deposition that will enable the development of nanostructures such as oxide quantum wires, and the use of PLD techniques that will allow the deposition of polymers and biomaterials such as MAPLE.

For further details on the technical content of the Meeting, see the following symposium summaries. Proceedings are available online at www.mrs.org.

\section{Amorphous and Nanocrystalline Silicon Shows Its Tricks}

Symposium A on Amorphous (a-) and Nanocrystalline Silicon-Based Films surveyed activities over a wide range of noncrystalline silicon, with many sessions on growth, and on applications such as solar cells, imagers, and TFTs.

R. Collins (PSU) described his use of evolutionary phase diagrams of amorphous and microcrystalline silicon in order to optimize the materials and the performance of their solar cells. This method emphasizes how the transitions between different regimes vary as the films become thicker.

H. Atwater (Caltech) summarized the status of work on nanocrystalline Si for optoelectronics and nonvolatile memories. The bandgap of nanocrystalline $\mathrm{Si}$ increases with decreasing grain size, but then the optical transition becomes limited by transitions at the surface $\mathrm{Si}=\mathrm{O}$ groups. Atwater said that Flash memory does not scale well, and there are competing memory systems with both much higher storage densities or shorter write times. To improve write times, the tunneling distance needs to be shortened, while retention time requires control of the energy profile of the tunnel barrier by using additional oxides.

J. Jang (Kyung Hee Univ., Korea) covered the various types of metal-induced crystallization of amorphous silicon. Ni, for example, can result in crystallization down

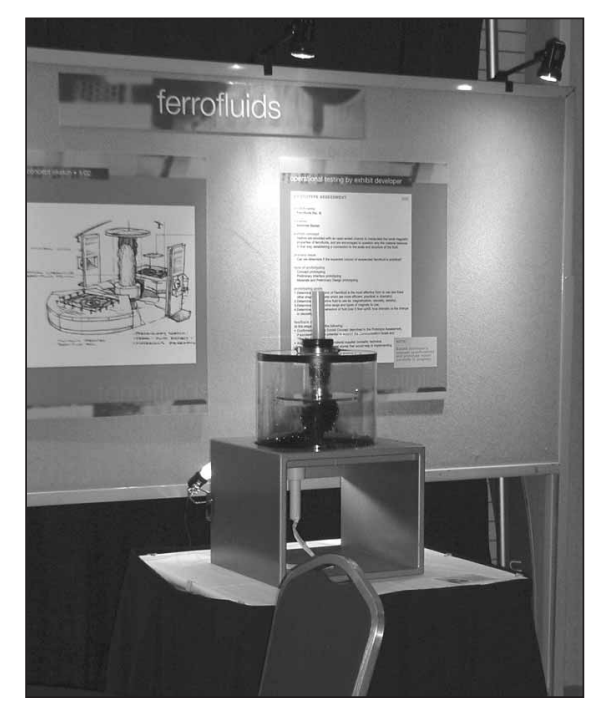

The ferrofluid display is part of the MRS science exhibit, Strange Matter, presented at the MRS meeting for input from attendees. More information on the exhibit is available at Web site www.strangematterexhibit.com.

to $300^{\circ} \mathrm{C}$ using the intermediate $\mathrm{NiSi}_{2}$ phase. The Ni gives rise to needle-shaped crystallites. Raising the substrate temperature to $550^{\circ} \mathrm{C}$ and controlling the amount of $\mathrm{Ni}$ used leads to very low nucleation densities, and to grain sizes of $40 \mu \mathrm{m}$. TFTs with hole mobility of $100 \mathrm{~cm}^{2} / \mathrm{Vs}$ were achieved.

R. Schropp (Univ. Utrecht) summarized the present state of the art of hotwire growth of a-Si and microcrystalline $\mathrm{Si}$. The advantages of hot wire for a-Si:H are the very high growth rates and good stability. Firms have now implemented commercial hot-wire systems with features such as automatic wire replacement to overcome its traditional limitations.

When using thermal CVD instead of plasma deposition, from precursors of disilane and silicon tetrafluoride, J. Hanna (Tokyo Inst. of Technology, Japan) was able to make microcrystalline Si TFTs with carrier mobilities of the order of $40 \mathrm{~cm}^{2} /$ Vs, which is about the highest for directly deposited material.

The symposium had many invited talks on solar cells. B. Rech (Institute of Photovoltaics, Germany) described the work on amorphous and microcrystalline stacked junction Si solar cells. Rech's group has achieved the highest conversion efficiencies of the order of $11.2 \%$. B. Yan (United Solar Corp., Michigan) covered work on single and multiple junction solar cells. The research team at United Solar Corp. is implementing microwave deposition to produce the microcrystalline Si. Q. Wang (NREL) showed how to optimize material choice and device design by using a combinatory approach to deposition under many different conditions in the same vacuum run.

D. Cohen (Univ. Oregon) described the photodegradation and defect generation processes in amorphous $\mathrm{Si}-\mathrm{Ge}$ alloys. He showed that this must occur by a local bond-switching process.

T. Ohmi (Tohoku Univ., Japan) showed the functioning of a microwave growth system with very low electron temperature for the production of $\mathrm{SiO}_{2}$ and silicon nitride films at $300^{\circ} \mathrm{C}$ with very low damage and metal impurity content. It functions as a remote plasma system, somewhat similar to those of Gerry Lucovsky (North Carolina State Univ.).

N. Mousseau (Univ. Montreal, Canada) described his work on the configuration changes in a-Si networks. The energies associated with these large changes were calculated. It was found that by far the most probable change is the bond switching interchange of the type previously denoted T2 by Wootten, Winer, and Weaire. He then described how this could be used to study the nucleation of microcrystalline-Si within large models of a-Si.

Symposium Support: BP Solar, MVS Systems, NREL, Sanyo Electric, United Solar Systems Corp., Voltaix, and Xerox-PARC.

\section{Polycrystalline Compound Semiconductor Photovoltaics Outperform Single-Crystal Counterparts (See MRS Proceedings Volume 763)}

Symposium B, Compound Semiconductor Photovoltaics, provided an interesting and dynamic overview of the central materials issues in, especially, Cuchalcopyrite and CdTe for thin-film solar cells. The overall perspective on compound semiconductors was widened by sessions on III-V materials and TCOs.

An engaging and inspiring discussion session on why small-grained polycrystalline solar cells work related many of the scientific contributions to one of the most intriguing scientific puzzles in this area. Cells made with polycrystalline $\mathrm{CdTe}$ and $\mathrm{Cu}(\mathrm{In}, \mathrm{Ga}) \mathrm{Se}_{2}$ significantly outperform their single-crystal counterparts. The properties of grain boundaries (GBs) in such cells were vigorously debated. Ideas such as bandgap widening due to ordered defects near the GBs and GB band bending coupled to an enhanced collection of minority carriers were discussed. Both ideas were supported by experimental results using advanced materials characterization techniques 
such as grazing angle XRD, TEM, and scanning probe microscopy.

Defects in photovoltaic materials were further characterized structurally with the use of EXAFS and related methods as well as by special electrical techniques such as transient photocapacitance spectroscopy. Contributions in the latter area, especially, are significantly advancing this field.

Many presentations concerned synthesis of devices and materials. The requirements of TCOs were also a major subject in the discussion session on whether polycrystalline thin-film tandem cells are a viable option for high efficiency. The conclusion drawn is that, if tandem cells are to beat single-junction thin-film cells which now approach $20 \%$ efficiency, very stringent demands will be put on all materials, not least of which, the TCOs. The complexity of this issue also came up during a discussion comparing requirements for high-yield, cheap, stable, and efficient cells for modules with those for record-efficiency laboratory cells.

Symposium Support: AFRL, NREL, ITN Energy Systems, Shell Solar Ind., and SULA Technologies.

\section{Applications Expand for Wide- Bandgap Semiconductors (See MRS Proceedings Volume 764)}

The theme of Symposium C was the numerous emerging applications for wide-bandgap semiconductors such as $\mathrm{GaN}, \mathrm{SiC}$, and $\mathrm{ZnO}$. While the use of these materials systems for visible light emitters is well established, there is now strong interest in $\mathrm{GaN} / \mathrm{AlGaN}$ transistors in power amplifiers for broad-band wireless communication systems. There is an interest in $\mathrm{SiC}$ and $\mathrm{GaN}$ for use in high-power conditioning and switching and also as high-temperature combustion-gas sensors. There is an interest in all of these materials for application in spintronic devices-in which the spin of the electron is exploited-in addition to the more conventional charge-based devices.

The tone of the symposium was set by two overview talks (L. Rea of WrightPatterson AFB, Ohio and H.B. Dietrich of ONR, Virginia) that covered the military interest in wide-bandgap materials and electronic devices in advanced hybridelectric vehicles, new generations of ships, and in aircraft avionics. Some of the challenges remaining before widespread commercialization and application of these devices were addressed in a series of talks covering recent results on device processing, device performance, and crystal growth (J.B. Casady, SemiSouth Labs., Mississippi; C.-M. Zetterling, , KTH, Royal Institute of Technology, Sweden; A.P.

\section{Graduate Students Receive Gold and Silver Awards}

During an awards ceremony held on April 23 at the 2003 Materials Research Society Spring Meeting, graduate student finalists received Gold and Silver Awards.

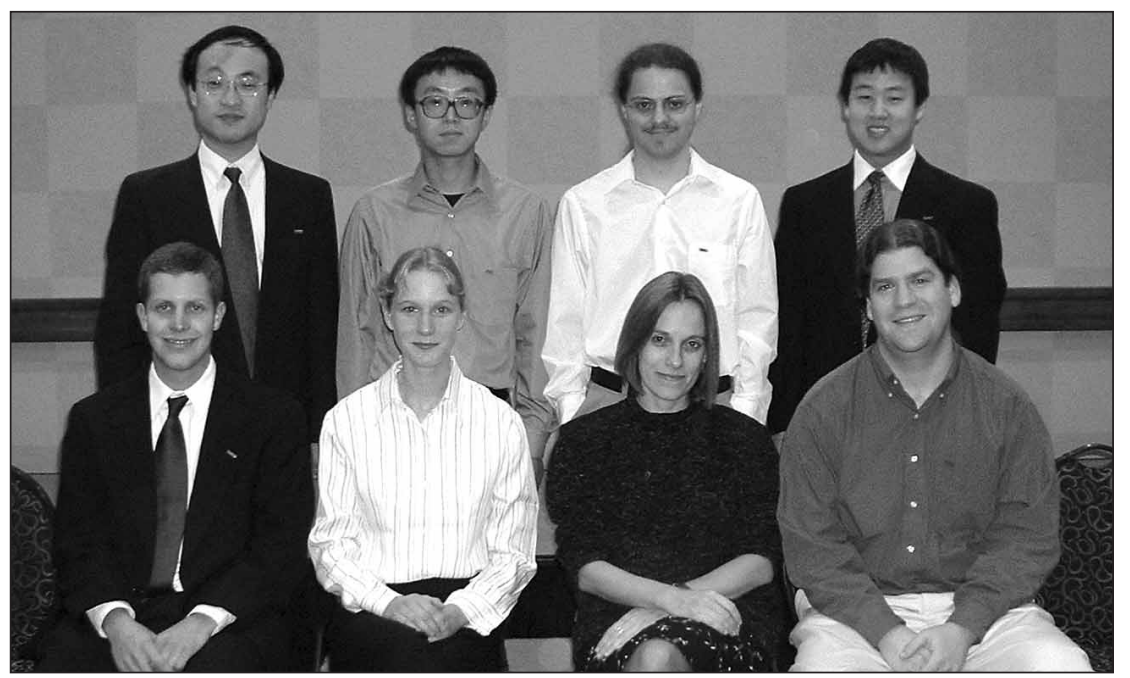

Graduate Student Gold Awards went to (first row, left to right) Jason B. Baxter (University of California-Santa Barbara), Cindi L. Dennis (University of Oxford, United Kingdom), Iris Visoly-Fisher (Weizmann Institute of Science, Israel), and Seth Coe (Massachusetts Institute of Technology); and (second row, left to right) Sen Liu (University of Washington), Liang-Shi Li (University of California-

Berkeley), Erich C. Walter (University of California-Irvine), and Minjoo "Larry" Lee (Massachusetts Institute of Technology).

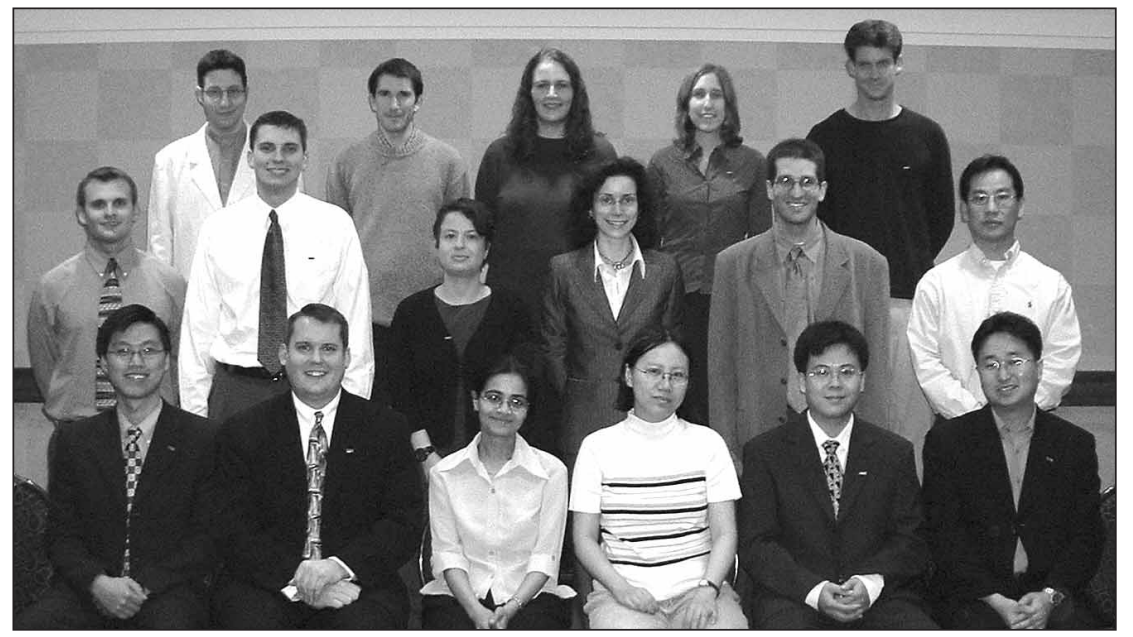

Graduate Student Silver Awards went to (first row, left to right) Chee Lip Gan (Singapore-Massachusetts Institute of Technology Alliance), Brandon Seal (Arizona State University), Prita Pant (Cornell University), Yulong Shen (Cornell University), Jiaxing Huang (University of California-Los Angeles), and Ki-Bum Lee (Northwestern University); (second row, left to right) Eric P. Guyer (Stanford University), James F. Hulvat (Northwestern University), Ruxandra Costescu (University of Illinois-Urbana-Champaign), Ana Filipa Nogueira Fixe (INESC-MN, Portugal), Eli D. Sone (Northwestern University), and Tae-Hee Lee (Georgia Institute of Technology); and (third row, left to right) Pavle Radovanovic (University of Washington), Olivier Noel (Université Haute Alsace, France), Terri L. Haskins (University of Rochester), Robin L. Hayes (University of California-Los Angeles), and Matthew Law (University of California-Berkeley). Not shown is Alexandra E. Porter (University of Cambridge, United Kingdom). 
Zhang, GE, New York; and J.S. Moon, HRL Labs, California).

A key aspect of the performance of $\mathrm{GaN} / \mathrm{AlGaN}$ devices is the need to passivate surface states that otherwise limit the output power at high drive voltages and to understand the effects of pre-process temperature stressing. These topics were reviewed by F. Ren (Univ. Florida) and M.J. Yannuzi (Wright-Patterson AFB). In an overview of the use of novel heterostructures for gas and liquid sensing, M. Stutzmann (WSI, Munich) described results for combustion-gas sensing and polar-liquid sensing that have application in many areas, including automobiles, space craft, and medicine. J.S. Williams (ANU, Australia), R.J. Shul (SNL, New Mexico), and I. Adesida (Univ. Illinois) discussed the many challenges in the processing of these materials, including the loss of one of the elements in the lattice during ion beam or plasma processing or annealing. Many novel phenomena such as changes in materials properties during electron injection can be exploited in device structures (L. Chernyak, Univ. Central Florida).

The emerging area of spintronics arises from the ability to make room-temperature ferromagnetism in wide-bandgap materials through the controlled introduction of transition metal ions such as Mn. Some of the key issues, such as retention of singlephase material and the identification of the mechanisms for the observed magnetic properties, were covered (Y.D. Park, Seoul Natl. Univ., Korea and J.M. Zavada, U.S. Army Research Office, North Carolina).

Symposium Support: Univ. of Florida.

\section{CMOS Front-End Scaling}

Faces Hurdles on the Road to

45-nm-Node Devices

(See MRS Proceedings Volume 765)

Symposium D on CMOS Front-End Materials and Process Technology addressed the most challenging roadblocks to scaling of CMOS devices to the 65-nm technology node and beyond. Highlights included an overview of the major achievements of the new high-к dielectrics by H. Iwai (Tokyo Inst. of Technology, Japan). Experimental results were presented with an effective oxide thickness of $6.5 \AA$ and $\times 10^{4}$ reduction in leakage current compared to the $\mathrm{SiO}_{2}$ equivalent.

V. Misra (NCSU) presented her group's work on selection of metal candidates for gate electrodes. This included alloying of materials as a novel approach to the challenges of dual metal gate integration.

Y.V. Ponomarev (Philips Research Leuven, Belgium) assessed the impact of these new materials and transistor architectures on the device and overall circuit performance. It was suggested that, for many applications, the problems associated with the current best high- $\kappa$ candidates might not be overcome in time for production of 45-nm node devices, although metal gates and/or radical transistor architectures might offer alternative solutions.

E.A. Fitzgerald (MIT) showed how to integrate III-V materials on Si using SiGe technology and K. Rim (IBM, New York) demonstrated the impact of strained Si upon the performance of deep-sub- $\mu \mathrm{m}$ CMOS devices. R. Huang (Univ. Texas) and G.M. Cohen (IBM, New York) both demonstrated novel approaches to achieving fully relaxed SiGe layers on SOI substrates.

R. Lindsay (IMEC, Belgium) compared various options for forming and activating junctions for the 45-nm node devices. Flash annealing and solid-phase regrowth were identified as the most likely candidates.

Symposium Support: Applied Materials, Axcelis Technologies, IBM T.J. Watson Research Center, Intel, Mattson Technology, Philips Semiconductors, TI, and Toshiba.

\section{Copper and Low- $\kappa$ Materials Perform on the CMP Scene (See MRS Proceedings Volume 767)}

The science of chemical-mechanical polishing (CMP) has come a long way since its development in the late 1980s. Gone are the days of normalized data presented on un-named, proprietary chemicals and arbitrary polish rate models. Researchers in Symposium F presented new and continued work to understand the fundamental removal mechanisms using chemistry, fluid dynamics, tribology, dimensionless analysis, and residence time distribution. In addition, many researchers presented process-related models and data, including refinement of planarization length determination, measurement of pad degradation, and the unique properties of ceria-based slurries.

The majority of talks were on copper and low- $\kappa$ polish research, as these are the least understood and the newest materials in CMP. Separate groups headed by F.M. Doyle and S.V. Babu, of Univ. California-Berkeley and Clarkson University, New York, are actively studying the chemistry and electrochemistry of copper polish slurries. P. Beaud (Swiss Federal Inst.), along with fellow researchers from France and Japan, studied polishing of low-א and Ta (barrier) materials. In situ detection of delamination using wavelet-based analysis of acoustic emissions was proposed by T.K. Das (Univ. South Florida). Other presentations discussed studies of post-CMP cleaning (A. Philipossian, Univ. Arizona), slurry viscosity as a function of shear rate (W. Lortz, Degussa AG, Germany) (see Figure), and Stribeck curve analysis (D.R. Evans, Sharp Laboratories of America).

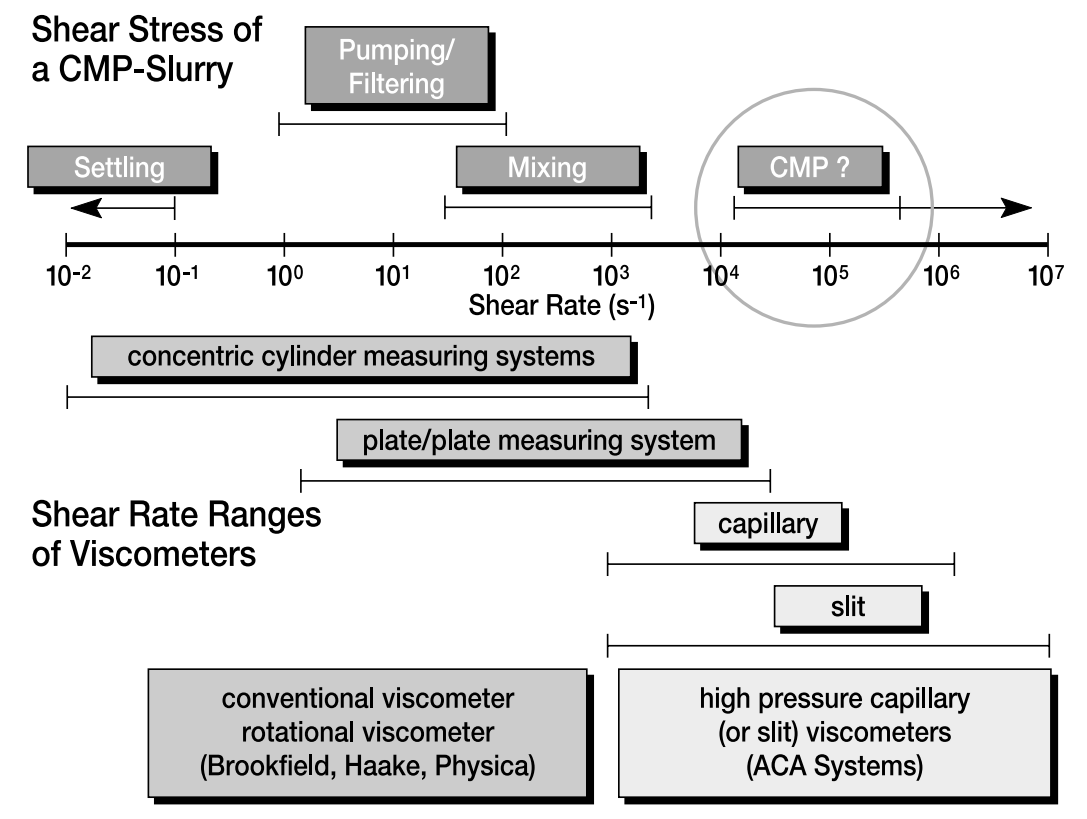

Figure. The shear rate during CMP is compared to other processes (Lortz). 


\section{Properties and Reliability of Interconnects and Low-Dielectric- Constant Materials Examined (See MRS Proceedings Volume 766)}

Symposium E on Materials, Technology, and Reliability for Advanced Interconnects and Low- $\kappa$ Dielectrics addressed a wide range of topics including electromigration and interconnect reliability, deposition and microstructure of metal films, novel interconnect concepts, and low- $\mathrm{K}$ materials and their properties.

In the metallization and reliability areas, J. Koike (Tohoku Univ.) proposed a new via pullout mechanism in $\mathrm{Cu}$ interconnects with an assumption of the presence of pre-existing voids and the possibility of void coalescence under initially large thermal tensile stress. He further suggested that good wettability at the copper/barrier interface was important in avoiding the via pullout. X. Lu from P. Ho's group at the University of Texas at Austin reported interfacial-diffusion-dominated mass transport for $\mathrm{Cu} / \mathrm{MSQ}$-low- $\mathrm{\kappa}$ materials that is the same as that in $\mathrm{Cu} / \mathrm{SiO}_{2}$.

In the area of low- $\kappa$ materials and their properties, several speakers reported progress toward improving the mechanical strength of spin-on and CVD-deposited carbon-doped oxides. H. Arao (Catalysts and Chemicals Industrial) reported on spin-on nano-clustering silica (NCS) materials having an elastic modulus, $E=10 \mathrm{GPa}$, an average pore size of $2 \mathrm{~nm}$, and a dielectric constant of $\kappa=2.3$. D.Y. Yoon (Seoul
Natl. Univ.) reported that incorporation of 1,2-bis(trimethoxysilyl)ethane (BTMSE) or dimethyldimethoxysilane (DMDMS) increased the mechanical strength of polysislesquioxanes (PSSQs) by four times, while maintaining a dielectric constant of $\kappa=2.7-2.8$. Although it has been demonstrated that CVD carbon-doped oxides can be extended to $\kappa<2.2$, there are still challenges in integrating these materials due to their weak mechanical strength ( 3 GPa).

Symposium Support: Applied Materials, ATMI, Dow Chemical, Honeywell Electronic Materials, Intel, TI, Thomas West, and Tokyo Electron America.

\section{Thin Films with Enhanced Function Integrated into Microsystems (See MRS Proceedings Volume 768)}

Symposium G addressed the science, engineering innovations, and applications of methods by which diverse thin-film materials and devices may be integrated for the purpose of enhancing the functionality and performance of microsystems. Topics ranged from heteroepitaxy to wafer bonding and layer transfer. The Symposium began with a joint session with Symposium D on "Heterogeneous Integration and Strained Si Technologies," featuring invited talks by E. Fitzgerald (MIT) and K. Rim (IBM). Speakers in this session highlighted progress in the understanding of the mechanics of compliant and graded heterostructures, and the implications of this new knowledge for the design and performance of devices

\section{Timothy J. Deming Delivers Outstanding Young Investigator Award Talk on Polypeptides}

The MRS Outstanding Young Investigator award recipient Timothy J. Deming (UCSB) gave his talk as part of Symposium $X$ about recent research on synthetic

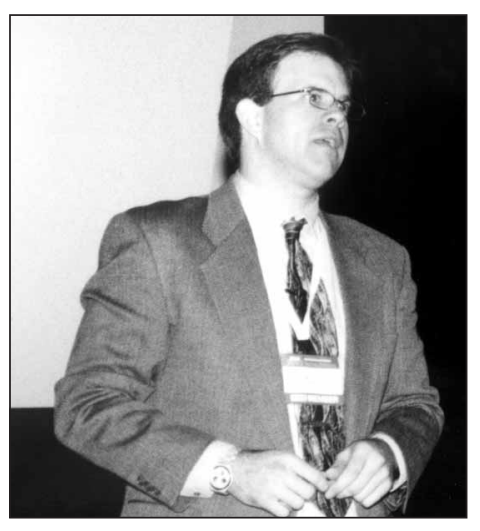
polypeptides. In a historic overview, he said that natural polypeptides were discovered in the early 1900s. Synthesis was achieved in the mid-20th century and synthetic polypeptides were used to correctly identify the $\alpha$-helix and $\beta$-sheet structures of these molecules. They are currently of great interest as synthetic materials made of biological components. Deming said that the aim of his group is to create synthetic mimics of protein materials. He discussed polymerization of these molecules using metal complexes. He also detailed self-assembly of these molecules in solution using various techniques and said that they provide "access to functional nanostructured materials of unrivaled complexi-

Timothy J. Deming of the

University of California-Santa

Barbara is the 2003 MRS

Outstanding Young Investigator. ty." Deming discussed the formation of vesicle structures from the polypeptides as an example of an application. Other applications include hydrogels and drug delivery. that exploit the properties of Si-Ge alloy heterostructures.

A session on wafer bonding was placed into context by U. Gösele (MPIHalle), who presented a comprehensive and insightful overview of the field. Invited speaker A. Brown (Duke) introduced the topic of heterogeneous integration for optoelectronics. S. Noda (Kyoto) discussed the application of wafer bonding and layer transfer to the assembly of intricate photonic crystals with performance that depends on a high degree of precision during the assembly process. Several speakers highlighted applications of bonding and layer transfer to the integration of Faraday rotators, LEDs, lasers, and piezoelectric device structures with microfluidics and high-speed electronics.

The final session of the Symposium dealt with advances and innovations in layer transfer. The repeated application of direct bonding and etch-back was shown by J. Burns (MIT) to be a promising approach to the assembly of 3D integrated circuits. The session concluded with a series of talks on ion cut and laser lift-off, two alternative approaches to layer transfer.

Symposium Support: ARO.

\section{Electronics Devices Show Their Flex (See MRS Proceedings Volume 769)}

The topic of Flexible ElectronicsMaterials and Device Technology was presented as Symposium H. The opening session laid the groundwork with talks on applications by D. Keys (DuPont Displays) and H. Becker (Covion Organic Semiconductors), funding opportunities by R. Reuss (DARPA), and technical challenges by S. Wagner (Princeton), G. Nisato (Philips), and J. Sheats (Rolltronics Corp.). These talks set the stage for four days of outstanding technical presentations covering subjects such as flexible substrates, TFTs, organic semiconductors, low-temperature silicon processing, and electronic textiles. A poster award went to I.-C. Cheng (Princeton) for a presentation on nanocrystalline silicon processing.

Symposium Support: DARPA.

\section{The Light Shines for Unconventional Optical Materials}

(See MRS Proceedings Volume 770)

Symposium I, Optoelectronics of GroupIV-Based Materials, kicked off with a tutorial session and ended with a discussion forum. In between, many exciting developments were uncovered, ranging from fundamental insight into light-emission mechanisms to major advances in device development and performance. 
An issue that provoked much interest was the origin of the luminescence emitted by $\mathrm{Si}$ nanocrystals embedded in $\mathrm{SiO}_{2}$. This has been an area of debate for more than a decade, but significant data was introduced at the meeting. In one important study, N. Daldosso and colleagues from INFM-Trento University used x-ray absorption spectroscopy in two distinct detection modes, total electron yield (TEY) and photoluminescence yield (PLY), to show that Si nanocrystals appear to be surrounded by a 1-nm thick shell of stressed $\mathrm{SiO}_{2}$ that participates in the luminescence mechanism. This added to a growing body of evidence for the role of the $\mathrm{Si} / \mathrm{SiO}_{2}$ interface in the luminescence process.

The observation of optical gain in silicon nanocrystal-based materials also remained a key issue, and insight was provided by Daldosso, J.-H. Ruan (Univ. Rochester), and N. Smith (ANU). A round-robin study by the first two groups demonstrated apparent gain in a particular class of samples but also highlighted the sensitivity of the measurement to the sample structure. Further collaborations were planned in an attempt to resolve this issue.

On the device front, several applications of group-IV semiconductors were highlighted, with emphasis on light-emitting structures and photodetectors. S. Coffa (STMicroelectronics) described developments in the fabrication of light-emitting devices based on Si-nanocrystal-sensitized rare-earth emission. These electroluminescent devices were shown to exhibit external quantum efficiencies as high as $10 \%$ and were being developed for applications such as on-chip, electrically isolated control of power devices.

Symposium Support: ARO.

\section{Structures and Metallic Materials of Photonic Crystals Explored}

At the beginning of Symposium J, a joint half-day session was held with Symposia $\mathrm{P}$ and $\mathrm{Q}$ as the topics related to microphotonics, nanophotonics, and photonic crystals. The program offered a special emphasis on structures with negative index of refraction (left-handed metamaterials and photonic crystals) and metallic materials with enhanced optical properties (plasmonics).

Among the highlights were presentations by the following invited speakers. S. Schultz (UCSD) discussed recent controversies related to artificial materials that exhibit a negative index of refraction. He discounted recent arguments that these materials were unphysical. An invited presentation from C. Soukoulis's group

\section{Poster Prizes Awarded at the 2003 MRS Spring Meeting}

The 2003 MRS Spring Meeting chairs awarded prizes for the best poster presentation. Prize recipients from poster sessions received $\$ 500$, a certificate, and the honor of having the winning poster displayed for the remainder of the Meeting. Posters awarded prizes and their authors were (D6.1) A New Type of Texture in Thin Films: NiSi, CoSi and -FeSi on Si(100), C. Detavernier, J.L. Jordan-Sweet, and C. Lavoie (IBM T.J. Watson Research, Yorktown Heights) and A. Özcan (Boston Univ.); (E3.28) Vapor Treatment of Copper Surface Using Organic Acids, K. Ishikawa, T. Yagishita, and M. Nakamura (Association of SuperAdvanced Electronics Technologies, Japan); (H6.8) Device Geometries for Thin Film Transistors of Nanocrystalline Silicon, I-C. Cheng and S. Wagner (Princeton); (L4.23) Blue Organic Electrophosphorescence Using Exothermic Host-Guest Energy Transfer, R.J. Holmes and S.R. Forrest (Princeton), Y.-J. Tung, R.C. Kwong, and J.J. Brown (Universal Display Corp., Ewing, N.J.), and S. Garon and M.E. Thompson (Univ. of Southern California, Los Angeles); (L10.6) Visible and $1.55 \mu \mathrm{m}$ Quantum Dot Organic Light Emitting Devices, S. Coe, V. Bulovic, J. Steckel, and M. Bawendi (MIT); (M9.3) Mechanics of Deformation of Single and Multiwall Carbon Nanotubes, A. Pantano, D.M. Parks, and M.C. Boyce (MIT); (O7.8) Amelogenin Induces Biomineralization at Specific pH, S. Habelitz, S.J. Marshall, M. Balooch, G.W. Marshall, W. Li, and P.K. DenBesten (UC-San Francisco); (P6.24) Self-Assembled, Intercalated, Responsive Polydiacetylene Based Crystals, X. Li, B. McCaughey, J. Pang, X.-L. Ji, and Y. Lu (Tulane Univ.); (R5.14) Investigation of Magnetic Microstructures Using Photoemission Electron Microscope, D.H. Wei and Y.J. Hsu (Synchrotron Radiation Research Center, Hsinchu, Taiwan), and J.Y. Ou and J.C. Wu (Natl. Changhua Univ. of Education, Changhua, Taiwan); and (Q5.20) Colloidal Template Method for Antireflecting Porous Surfaces, H. Young Koo, D.K. Yi, S.J. Yoo, H.S. Shim, and D.Y. Kim (Kwang-Ju Inst. of Science and Technology, Kwang-Ju, Republic of Korea).

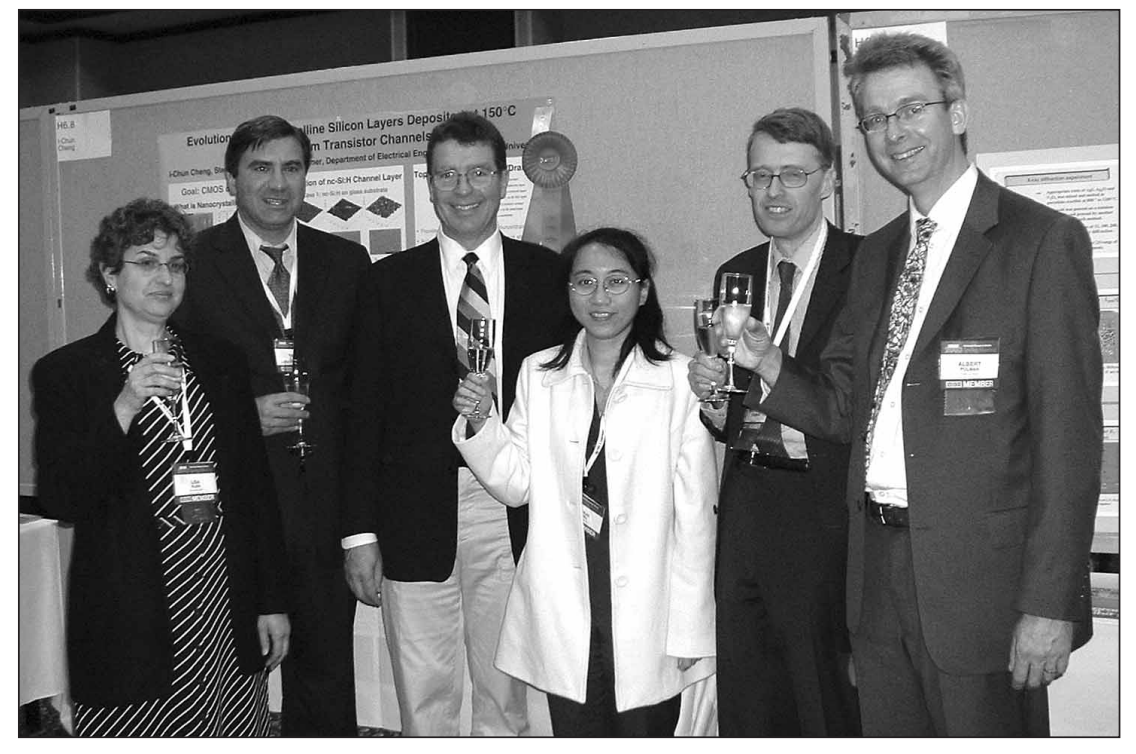

(Iowa State) addressed the possibility of using photonic crystals as a negative refractive index material.

J. Joannopoulos (MIT) described the effects of propagating shock waves through photonic bandgap crystals. He showed theoretically that this would allow frequency conversion, pulse compression, and other useful and interesting phenomena. N. Venkataraman (Corning) discussed work on photonic bandgap fibers that exhibit losses of $13 \mathrm{~dB} / \mathrm{km}$. He discussed the theoretical loss of $0.02 \mathrm{~dB} / \mathrm{km}$ and possible future strategies to further decrease experimental losses.

A. Scherer (Caltech) discussed the combination of photonic crystals with microfluidic devices. He addressed the possibility of utilizing defects in 2D photonic crystals as a sensitive sensor environment for microfluidics.

S. Akiyama, in a contributed talk from 


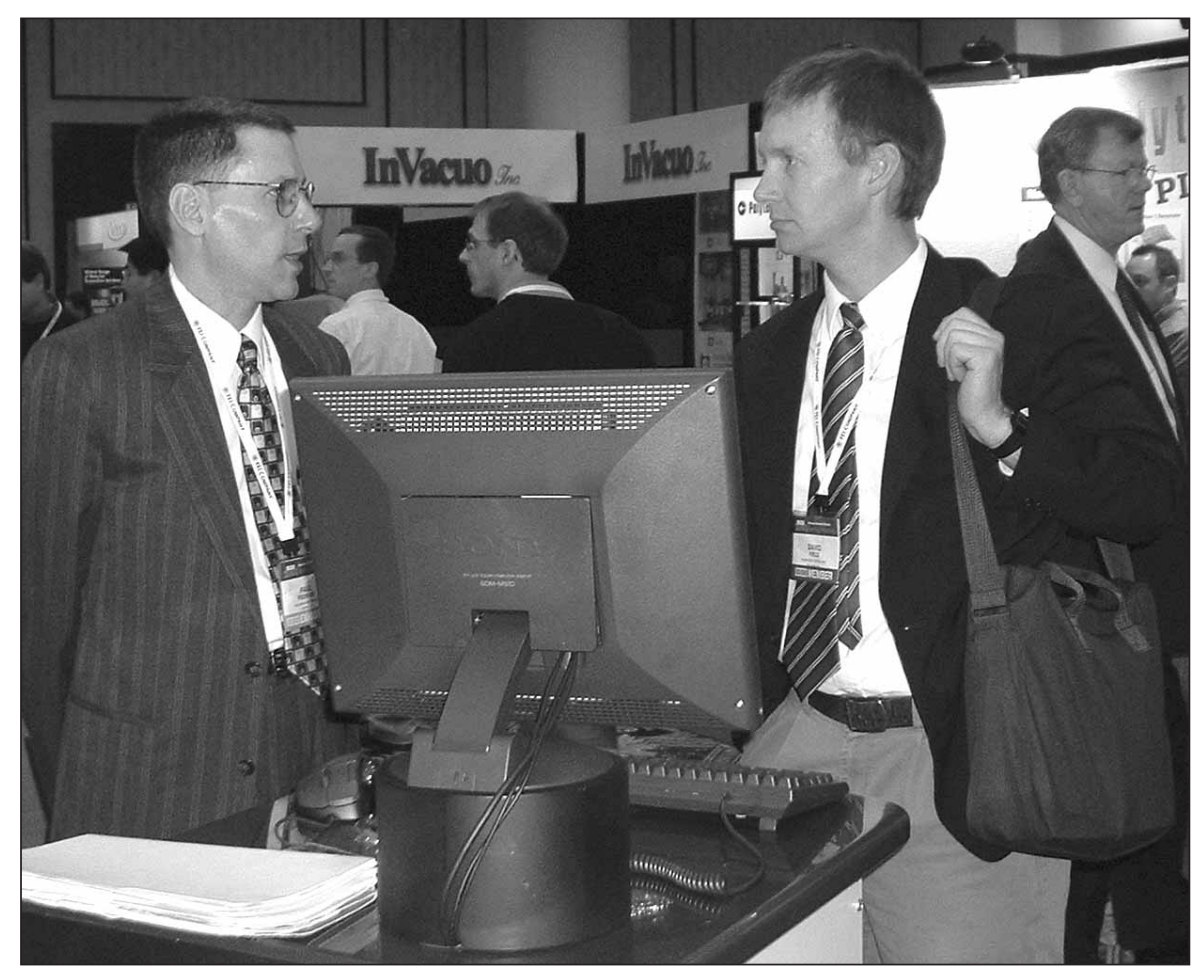

The MRS equipment exhibit featured over 90 exhibitors from around the world, displaying a range of equipment, services, products, publications, and software.

L.C. Kimerling's group (MIT), described the use of air trenches as a simple way to make sharp turns in an optical waveguide. Akiyama explained experiments that combined the advantages of low index contrast waveguides (low transmission losses) with the addition of high index contrast at locations where a sharp turn was required. At these regions, an air trench was etched on each side of the waveguide to facilitate the turn. The fabrication procedure for this strategy was both simple and realizable.

Symposium Support: DARPA, Elsevier, and 3M.

\section{Characterization of Molecular-Scale Electronics Leads to New Pathways}

In Symposium K on Molecular-Scale Electronics and Optoelectronics, a series of novel ideas and concepts has been presented regarding the characterization of molecular devices, their transport properties, and the integration of molecular electronics with silicon technology. An important aspect that has been studied at length has been the structural properties of molecular structures in contact with different metals. For instance, it has been shown that no degradation or reorientation of selfassembled monolayers occurs during gold or titanium deposition. It was also report- ed that conjugated dithiols prefer a vertical, not flat, configuration with respect to the metal surface. In addition, detailed studies of the electrode formation and structure indicate that strong Coulomb correlations can develop in nanoscale contacts, leading to suppression of the density of states at the Fermi level. These issues are important to understand the charge transport mechanism in molecular devices.

In the same vein, studies of transport properties of conjugated molecules suggest that the electron transfer process is ruled by local dipoles and potentials forming inside the molecule, allowing a large phase space of possible structures with different current-voltage characteristics. Several studies have focused on combining molecular electronics with silicon technology, a promising avenue for commercialization of molecular devices. There is both experimental and theoretical evidence of negative differential resistance (NDR) in systems comprising organic molecules on silicon surfaces. Very large on/off ratios have been reported, suggesting that inelastic phonon scattering, usually responsible for the degradation of NDR in solid-state devices, be considerably suppressed in these hybrid devices.

Symposium Support: Luna Innovations.

\section{Organic Materials Are on Displays (See MRS Proceedings Volume 771)}

Symposium L on Organic and Polymeric Materials and Devices provided an overview of recent developments in this emerging field. Although already commercialized, OLEDs are still extensively studied. New results on the understanding of loss processes and novel device concepts such as charge-generation layers, metal complexes, and the use of heterojunctions were presented and are leading to further improvement in efficiency.

In the field of organic transistors, major progress was reported on the charge transport properties of organic semiconductors, and mobilities of up to $5 \mathrm{~cm}^{2} / \mathrm{Vs}$ have been reported for pentacene-based transistors. Furthermore, high-mobility $n$-type materials have been reported, which enable the development of ambipolar organic electronic circuits. In the photovoltaic applications, the efficiency of polymer $/ \mathrm{C}_{60}$-based solar cells has been improved to $3 \%$ under AM 1.5 conditions, enabled by the use of a new fullerenebased light-absorbing acceptor. Also, some approaches to make fully printable displays on substrates such as textiles and paper were introduced, which might open a way to new applications of organic optoelectronic devices.

Symposium Support: Cambridge Display Technology and Plastic Logic.

\section{Carbon Nanotubes Move Toward \\ Uses in Biology, Field-Emission \\ Devices, and Strong Fibers \\ (See MRS Proceedings Volume 772)}

Symposium M, Nanotube-Based Devices, was devoted to the broad definition of "device" applications that have emerged for carbon nanotubes (CNTs) most recently. The session introduced developments ranging from large-scale synthesis of CNT arrays to nanotube transistors, but with the focus squarely on how these developments might transition to technology.

An emerging theme in the session was biologically based or biologically inspired applications of CNTs. A. Dalton (Univ. Texas-Dallas) and co-workers have shown possible routes for the organization of CNTs using proteins while A. Jagota (DuPont) and co-workers have attached peptides to nanotubes in the hope that they may one day be used to select specific tube chiralities. Development on nanotube- and nanobelt-based sensor systems was presented by several researchers. Most notably, the use of nanocantilevers as sensors by Z.L Wang (Georgia Inst. of Technology) and the separate works on chemical and gas-sensing 


\section{Government Funding Seminars Highlight Emphasis in Materials Research}

The 10th in a series of government materials research funding seminars was presented at the 2003 MRS Spring Meeting, featuring representatives from the Air Force Office of Scientific Research (AFOSR), the Defense Advanced Research Projects Agency (DARPA), the Department of Energy (DOE), and the National Science Foundation (NSF). In its debut seminar at an MRS Meeting, the structure of AFOSR was outlined by director Lyle Schwartz. He also identified the various program managers who deal with funding materials research. Examples of basic research contributions include self-healing polymer composites, which provide long life for structures and materials for air and space; poly-nitrogen, which provides high-density energy storage for propulsion and/or combustion; and dip-pen nanolithography, which reduces the weight of electronic components for use in air and space craft. Schwartz described six themes that have been identified for enhanced funding: cooperative control, plasma dynamics, miniaturization science for space, biologically inspired concepts, type II quantum computation, and materials engineering. Almost all of these are relevant to materials researchers. Schwartz also introduced a new program called MEANS-Materials Engineering for Affordable New Systems-whose mission is to exploit computational materials science and engineering to develop techniques for coupling models of materials behavior.

Steven Wax, the deputy director of the Defense Sciences Office and primarily responsible for materials-related programs at DARPA, said that research programs at his agency tend to be flexible. DARPA differs from other funding agencies, he said, in that it is project- and theme-oriented and is driven by the program manager. For a proposal to be successfully funded, there needs to be a high-risk paradigm shift from the Department of Defense perspective and it must have a champion, which means someone within DARPA who strongly endorses a project or someone external who is well known in the field. Wax described a research project on structural amorphous metals as an example. The research also needs to be very interdisciplinary, Wax said, offering projects on bioderived and biochemical materials as examples. To become involved, Wax

\section{Government Funding Information in Materials Research}

Air Force Office of Scientific Research (AFOSR) www.afosr.af.mil

Defense Advanced Research Projects Agency (DARPA) www.darpa.mil

\section{Department of Energy (DOE)}

www.eere.energy.gov/hydrogenandfuelcells; www.science. doe.gov/bes/

National Science Foundation (NSF)

www.nsf.gov suggested that researchers work with a program manager and find a champion. He also suggested that more senior researchers become program managers themselves.

Tom Weber, director of the Division of Materials Research (DMR) of NSF, covered what is new in funding in the division, continuing a series of similar seminars held during previous MRS meetings. With the caveat that the FY 2003 budget was not yet available to him, and with the FY 2004 budget still uncertain, Weber summarized funding trends and program plans. DMR con-

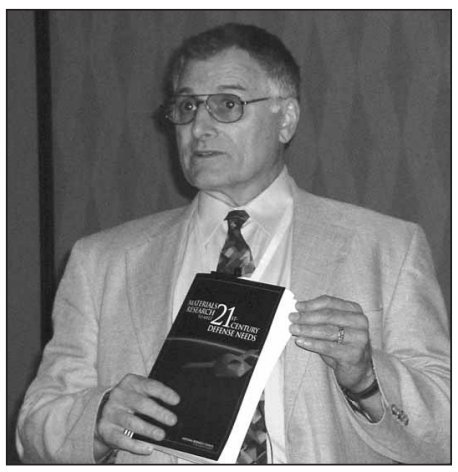

Lyle Schwartz, director of the U.S. Air Force Office of Scientific Research, refers to NMAB's report, "Materials Research to Meet 21st Century Defense Needs," in guiding funding priorities. tinues to extend programs aimed at international collaboration, with active efforts in Europe (even outside the European Commission funding arenas) and the Americas, and with budding collaborations initiated in Africa, India, Russia, and the Near East. On the instrumentation side, to fill the gap between smaller NSF Middleware Initiative projects capped at \$2 million and large construction projects in the $\$ 100$ million range, a program solicitation is planned for 2004 to fund midscale instrumentation. Nanotechnology continues to be a key focus as it is the primary growth area in an otherwise historically flat budget. Weber concluded by suggesting two actions for materials researchers to help ensure funding for the future. First, he said, get the word out that materials research is one of the physical sciences, so that it is not left behind; and second, tell NSF about good results.

A seminar was conducted by two representatives of DOE, John Petrovic, technology development manager of Hydrogen and Fuel Cells and Jane Zhu, program manager of the Division of Materials Sciences and Engineering. Petrovic concentrated specifically on the FreedomCAR (cooperative automotive research) program and the hydrogen fuel cell program. He said that R\&D efforts are encouraged for tanks in which compressed and liquid hydrogen is stored, complex metal hydrides, carbon-based storage, chemical storage, and other advanced concepts. Zhu described future research themes in basic energy sciences that include realization of the nanoscale revolution, complex systems, advanced computing, and "seeing" atoms. She recommended a recent report, "Basic Energy Needs to Assure a Secure Energy Future," available online at www.science.doe.gov/bes/BESAC/reports.html. utilizing nanotubes by A. Auvray (CEAMotorola), Y. Song (Wayne State), J. Li (NASA-Ames), and C.H.P. Poa (Univ. Surrey), all represent significant progress in sensor technology within the last year. These novel developments suggest that nanotube-based technology may eventual- ly well reach into biomedical applications.

Significant advances have also been made in field-emission device technology based on nanotubes. As demonstrated by a consortium of co-workers headed by Thales Research in France, large-area field-emission device arrays have possible applications in more than just flat-panel displays, but also as high-density lithography systems and triode amplifiers. Several different groups have now shown astonishing control over placement of field emitters within nanolithographed silicon-oxide-metal structures, represent- 
ing a major improvement in CNT-silicon hybrid integration techniques.

CNT transistors continue to evolve into more complex structures and functionalities as evident from work by the groups at IBM Research and the Max-PlanckInstitut in Stuttgart, as well as others. Of particular note are developments in the use of nonlinear optical properties in CNTs for high-power-laser optical elements as discussed by Y. Sakakibara
(AIST) and co-workers.

Exciting results on the creation of nanotube-based fibers was presented by $\mathrm{R}$. Baughman and co-workers at the University of Texas-Dallas. Long thought to be ideal reinforcing fibers for an organic matrix, nanotubes have yet to realize widespread use in such applications. Primarily due to issues of pullout and matrix adherence, many materials do not show enhanced mechanical properties

Biology Meets Micro- and Nanotechnology (See MRS Proceedings Volume 773)

Symposium $\mathrm{N}$ on biomicroelectromechanical systems (bioMEMS) provided a broad overview of various methods whereby conventional biological tools are integrated with micro- or nanosystems. The integration leads to the development of novel devices for a variety of clinical applications.

In the area of drug delivery and therapeutics, new devices and techniques based on various materials from silicon to carbon nanotubes were discussed. M. Madou (UC-Irvine) described a novel drug delivery technology based on artificial muscle and smart sensors. H. Gao (MPI-Metals Research, Stuttgart) presented moleculardynamics simulations for DNA insertion into nanotubes which has applications in molecular electronics and gene delivery systems (see Figure). J. Kornfield (Caltech) provided some insight into creating and manipulating intraocular lenses developed from biopolymers.
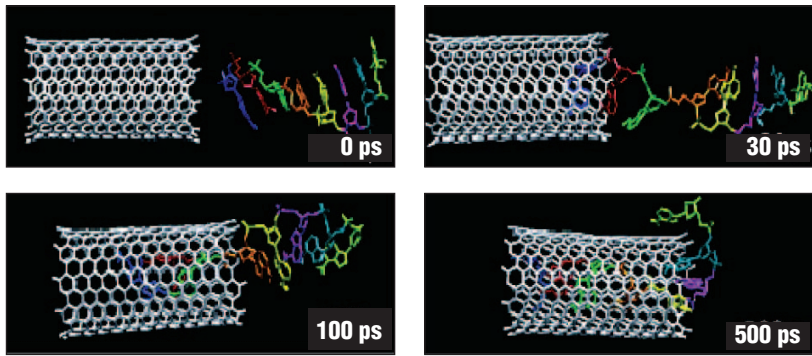

Figure. Simulation snapshots of a DNA oligonucleotide interacting with a carbon nanotube.

The session on tissue engineering focused on techniques of patterning a wide variety of cell types on biocompatible substrates using optical and electrical methodologies. S. Esener (UC-San Diego) described an optical technique for cell sorting which has been commercialized by Genoptix ${ }^{\mathrm{TM}}$ in San Diego. T. Desai (Boston Univ.) shared her knowledge pertaining to the development of tissue scaffolds for cardiovascular applications.

In the session on bio-nanosystems, M. Sarikaya (Univ. Washington) presented the latest developments in self-assembly of nanostructures based on genetically engineered proteins, and L. Lee (UC-Berkeley) described the current state of the art in biophotonic MEMS devices.

The session on biosensing devices gave an in-depth perspective into designing a variety of sensing devices based on amorphous carbon, polycrystalline diamond, and porous silicon platforms. Student researchers from the University of California, Riverside, supervised by C.S. Ozkan and M. Ozkan, discussed the development of single-cell-based sensors potentially capable of being applied to real-time scenarios as "detect-to-warn" systems.

The Symposium gave an overall perspective about the technology available and the requirements for taking a leap from bioMEMS toward bio-nanotechnology.

Symposium Support: ALZA Corp., Analog Devices, IDG Ventures, Micralyne, MEMS Ind. Grp., and MicroCHIPS.

when nanotubes are dispersed into them. Baughman's team seems to have finally overcome this difficulty with fibers that exceed the toughness of spider silk by several times. It should be noted that while it took nature 400 million years to develop spider silk, it took Baughman and his team only two years.

Symposium Support: CarboLex, Clemson Univ.The Center for Optical MS, Clemson Univ.-School of MSEE, IBM Research Division, ILJIN Nanotech, Michelin Americas Research and Development Center, Nanoledge SA, RHK Technology, and Toyota Motor Europe.

\section{Biology Folds Itself into Materials Developments}

\section{(See MRS Proceedings Volume 774)}

Symposium $\mathrm{O}$ on bio-inspired materials presented talks on a broad expanse of biomaterials, including presentations on polymeric and amphiphilic biomaterials, hard-tissue biomaterials, and biomimetically nanostructured materials. One recurring topic addressed the importance of interfaces and boundaries in organizing biomolecules. D. Talham (Univ. Florida) presented work on the nucleation of crystal growth at phospholipid monolayers. J. van Hest (Nijmegen Univ.) touched on this theme in describing a novel lipid-modified polypeptide his group designed, which only folds properly (to a beta-hairpin turn) when both ends are anchored into a bilayer membrane. J. Aizenberg (Bell Labs, Lucent Tech) showed enhanced crystal growth in microstructured arrays containing "microsumps" for material excluded by the crystallization process.

A second key theme was the creation of novel cellular environments. S. Rizzi (Univ. Zurich, ETH), working with J. Hubbell (Univ. Zurich, ETH), described a synthetic extracellular matrix that could be remodeled by active cellular processes, not simply degraded by way of generic hydrolysis reactions. H. Baca (Univ. New Mexico), with C.J. Brinker (Univ. New Mexico, SNL), presented work on entrapping living cells in silica as a means of creating robust biosensors for pathogens or chemical agents.

Several talks demonstrated how nature can be improved upon. Tirrell (Caltech) described methods to engineer bacterial cells that permit the biosynthesis of novel protein polymers that contain any of dozens of non-natural amino acids, many of which add functionality not found in natural proteins. A. Belcher (MIT) described the directed evolution of peptides, using phage display, which recognize specific crystal surfaces and thus control growth and morphology. R. Naik and M. Stone of AFRL isolated phages 
that bind inorganic surfaces very strongly, and then exploited the phage surface peptides for the synthesis of novel inorganic materials.

Symposium Support: Boston Scientific Corp., NIH/National Inst. of Biomedical Imaging and Bioengineering, NIG National Inst. of Dental and Craniofacial Research, AFRL, Avanti Polar Lipids, DuPont Central RED, and NSF.

\section{Advances Seen in Self-Assembled Nanostructured Materials (See MRS Proceedings Volume 775)}

The method of self-assembly that spontaneously assembles and organizes various building blocks into hierarchical structures through noncovalent interactions has emerged as one of the most promising techniques for productive fabrication of nanostructured materials. Symposium $P$ on Self-Assembled Nanostructured Materials highlighted major advancements in this rapidly growing field.

Advancements in inorganic mesostructured materials were made possible with the fundamental understanding of the evaporation-induced self-assembly process using in situ x-ray scattering or diffraction techniques, functionalization of the meso-structures with temperaturesensitive or photo-responsive components, controlled growth and morphology of the mesostructured materials using interfacial phenomena, novel assembly of semiconductor clusters, and with the applications of the mesostructures such as the use of mesoporous titania for photovoltaics and of mesoporous silica templates to synthesize metal, semiconductor, or carbon mesostructures.

Design and fabrication of self-assembled polymeric systems with spatially defined functionalities and mesoscale structures produced a major theme of this Symposium. Advancements in these systems occurred by the development of electricfield-responsive self-assembled liquidcrystalline systems; shear-aligned selfassembled anisotropic ionic-conducting block copolymers; and by the fabrication of conjugated, intercalated, responsive polydiacetylene systems. These advancements hold potential applications in sensor, battery, optics, and other applications.

Another important theme was the construction of hierarchical structures using nanoparticles, nanowires, or other building blocks. Highlights include the assembly of core-shelled nanoparticles into photonic crystals, nanowires and nanorods into thin films, and the assembly of diamondoids into higher diamondoid crystals. Researchers at ChevronTexaco recently isolated diamondoids containing 4-11 diamondcrystal cages from petroleum and recrystallized them into crystals. Rigidity, strength, remarkable assortments of shapes-including resolvable chiral forms-and multiple, readily derivatizable attachment sites make them valuable nanoscale molecular blocks for the synthesis of many advanced materials.

\section{From Smart Dust to Nanorods Nanostructures Gain Interest (See MRS Proceedings Volume 776)}

Nanostructures have attracted steadily growing interest due to their peculiar, fascinating properties, and unique applications superior to the bulk materials. The ability to generate such minuscule structures is central to the advance of essentially every area in modern science and technology (e.g., electronics, photonics, and information storage). Although nanostructures on the scale of tens of nanometers can be conveniently fabricated using advanced nanolithographic techniques such as e-beam writing, the development of these methods into practical routes to large numbers of nanostructures rapidly and at low-cost still requires great ingenuity. Unconventional techniques (or the so-called "bottom-up approach") may provide a more promising strategy for the formation of nanostructures in terms of cost, throughput, and potential for large-scale production. Symposium $Q$ focused on three different themes related to nanostructures: (1) unconventional approaches to the synthesis of nanostructures with low-dimensional morphologies; (2) self-assembly of such nanostructures into functional architectures, devices, and systems; and (3) novel

\section{Frontiers of Silicon, Bio-Inspired, and Nanocrystal Research Form Core of Lunchtime Reviews}

Symposium X on Frontiers of Materials Research offered a mixture of technical topics and issues related to materials research. Among the technical talks was a presentation by Meikei Ieong (IBM T.J. Watson Research Center, Yorktown Heights) on high-performance silicon device technology. He said that a number of innovations in CMOS have allowed for continuing scaling down of gate lengths, which has enabled the computational power of silicon devices to double every year. Ieong said that there are limits to the scaling down of the SOI layer and new materials and structures are under development to overcome these obstacles, such as 3D integration of multiple layers of transistors.

Mark Alper (LBNL) presented a list of relevant items that biology has to offer materials researchers. These include biological molecular machines-for example, a virus that can compactly pack DNA inside withstanding a pressure of $60 \mathrm{~atm}-$ enzymes, membranes, and sugar molecules. While biology has been a descriptive science until recently, Alper said, it can now be viewed more as a toolkit. Time will tell whether biology can be the basis for actively used materials and devices, he said.

Paul Alivisatos (UC-Berkeley) addressed nanocrystal science and technology. In particular, he discussed the synthesis and applications of nanometer-sized inorganic solid crystals that exhibit predictable physical and chemical properties, which are strongly size-dependent. Alivisatos said that it has now become feasible to precisely control the size and shapes of the nanocrystals. Among application opportunities, he detailed an example of a solar cell in which self-assembled nanorods, made from materials such as CdSe, are embedded in a polymer. Fabrication does not require vacuum and is relatively simple. Alivisatos also discussed CdSe tetrapods, which could be the inorganic equivalent of dendrimers.

In other issues related to materials research, materials scientists and engineers Timothy Hsieh, Jonathan Hack, and David M. Longo, from the intellectual property law firm of Finnegan, Henderson, Farabow, Garrett \& Dunner, L.L.P., overviewed the patent application process. They presented a case study, following a hypothetical nanotechnology invention. They explained benefits of having a patent, the importance of documenting the invention, and various issues relating to publishing and selling the invention.

Sylvia Johnson (NASA Ames Research Center) reported on the outcome of a workshop on workforce and education issues held in Irvine, California, on October 21, 2002. The workshop indicated that it is unknown whether the United States has the right number, mix, and quality of materials scientists and engineers to meet current and future demands. Johnson said that the discipline of materials science and engineering is highly fragmented and difficult to define. The identity issue needs to be resolved, she said, and the educational curriculum also needs to be redefined and optimized. 
concepts in designing nanoscale electronic and photonic devices. The Symposium offered sessions on quantum dots, nanoparticles, nanowires, self-assembly, photonic crystals, and molecular electronics and photonics. This topic stimulated interactions between researchers from a broad range of areas as a joint session was held with Symposia J and P on self-assembly.

L. Brus (Columbia) described some recent studies related to the electronic structures of 1-2 nm silicon nanocrystals whose surfaces are passivated with oxide shells and hydrogen atoms. M. Sailor (UCSD) discussed how nanoparticles made of porous silicon (called "smart dust") could be encoded with photonic features and then used to detect toxic chemicals and biological species. D. Norris (Univ. Minnesota) described a simple and convenient method for derivatizing quantum dots with hydrophilic lipids, as well as their use as fluorescent labels in biological systems. X. Peng (Univ. Arkansas) explicitly discussed the rational synthesis of semiconductor nanocrystals with controllable sizes and aspect-ratios. C. Murray (IBM) presented a solution-phase method for the synthesis of PbSe nanocrystals and nanowires. R. Vaia discussed a range of research activities at the AFRL related to the fabrication of organic-inorganic hybrid photonic systems. V. Colvin (Rice Univ., California) illustrated how biological nanostructures such as viruses could be used as templates to generate 3D metal nanostructures.

In the joint session, G. Stucky (UCSB) demonstrated how self-assembly on different scales could be exploited to create nanostructures for various applications. P. Wiltzius (Univ. Illinois) discussed the use of self-assembly (with spherical colloids as the building blocks) and twophoton optical lithography in generating photonic crystals.

C. Murphy (Univ. South Carolina) discussed a seed-mediated method for the synthesis of gold and silver nanorods with controllable aspect ratios. W. Buhro (Washington Univ., Missouri) presented recent studies on the synthesis and optical characterization of quantum wires made of III-V and II-VI semiconductors. N. Abbott (Univ. Wisconsin-Madison) discussed how liquid crystals could be used as an amplifying system for detecting biological species. S.A. Jenekhe (Univ. Washington, Washington) described the rational design and self-assembly of conjugated polymers into uniform nanowires. J. Rogers (Bell Laboratories / Lucent Technologies, New Jersey) discussed a number of methods for fabricating electronic devices from soft organic

\section{Nanomagnetism Pushes the Limits of Site and Characterization Scales}

Symposium R focused on "pushing the limits" in the field of nanomagnetism, making structures smaller (nanoscale) and characterizing them with increased accuracy and decreased measurement time scales (femtosecond range).

Nanostructured magnets with controlled sizes have been prepared using electron beams, porous alumina (K. Nielsch, MIT and I. Roshchin, UCSD), block copolymers (M. Tuominen, Univ. Massachusetts and C.A. Ross, MIT), scanning probe lithography (C. Van Haesendonck, Katholieke Univ. Leuven, Belgium), and laser interferometry (Ross). This makes artificially prepared nanomagnets extremely useful for studies of magnetism in confined geometries and hold promise in a variety of applications in the storage and sensing technologies (J. De Boeck, IMEC, Belgium). Examples of some of the most representative techniques for the preparation of nanomagnets are shown in the figure.

Characterization of nanostructured materials is a challenging endeavor. E.D. Dahlberg (Univ. Minnesota) described difficulties that occur using MFM and argued that these techniques must be combined with quantitative micromagnetic calculations. TEM can determine detailed magnetic structures at the nanoscale level (J. Chapman, Univ. Glasgow). The use of reciprocal space techniques such as neutron scattering and synchrotron radiation are at a stage where quantitative measurements can be obtained from small quantities of material (S. Sinha, UCSD and B.M. Barnes, Univ. Wisconsin-Madison). Several novel synchrotron-based techniques have been developed which allow element-specific characterization (J. Stöhr, Stanford and J.-P. Locquet, IBM-Switzerland). A novel first-order-reversalcurve method (K. Liu from UC-Davis) gives extremely detailed information on the reversal process of nanomagnets.

Nanostructured magnets exhibit physical phenomena that were unexpected by simple models or micromagnetic calculations. These include the development of vortex states due to the interplay between spatial confinement and magnetic energetics, modification of reversal processes in exchange-biased nanomagnets (I.V. Roshchin, UCSD), and unusual magnetic states in square (M. Grimsditch, Argonne) and interacting rings (V. Metlushko, Univ. Illinois-Chicago). The search for enhanced ballistic magnetoresistance has given negative results in lithographed Ni (A. Lukaszew, Univ. Toledo) and Co (M.I. Montero, UCSD) nanobridges. Nanostructured magnetic tunnel junctions show interesting magnetic imprinting effects (J.L. Costa-Krämer, CNM-CSIC, Spain). New magnetic materials have been discovered using fullerenes as the basic building blocks (K.V. Rao, Royal Inst. of Technology, Sweden).
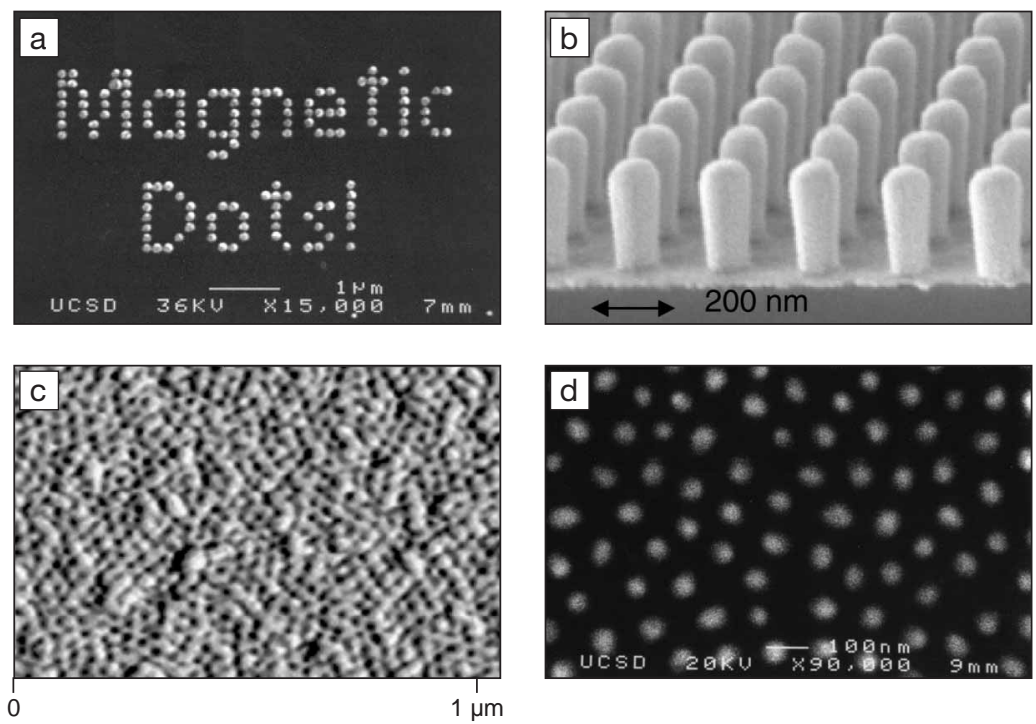

Figure. Nanolithography methods: (a) electron beam; (b) laser interference; (c) diblock copolymer; and (d) alumina nanopores. Prepared by I.V. Roshchin. Images provided by A. Hoffmann, C. Ross, K. Liu, C.P. Li, and I.V. Roshchin. 
materials. R. Penner (UC-Irvine) discussed the use of metal nanowires in chemical sensing.

Symposium Support: 3M, CW Group, Duke Scientific Corp., GE, Industrial Technology Research Inst., and Nanomix.

\section{Thermal Transport Follows Interesting Paths on the Nanoscale}

Symposium S, Nanoscale Thermal Transport-From Fundamentals to Devices, focused on the issue of heat transport in nanostructures. For such systems, the classical picture of a diffusive heat flow mechanism is often not applicable because the phonons or electrons that carry heat have mean free paths comparable to or smaller than the nanoscale feature size. W. Ford (Intel, Oregon) and K.E. Goodson (Stanford) discussed chal-

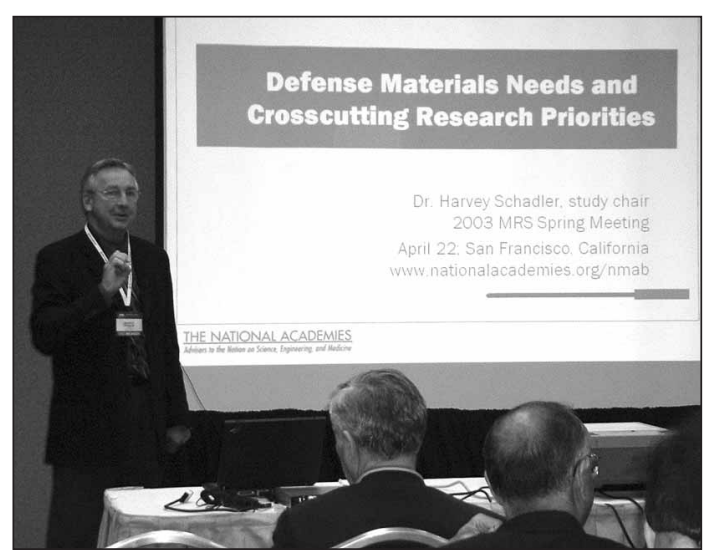

Harvey Schadler (retired from General Electric) gives an overview of the National Materials Advisory Board's report on materials research and defense needs.

crosscutting research priorities. Harry Lipsitt (Wright State materials and processes used for structural and multifunctional materials. John Gassner (Natrick Soldier Center) examined materials for energy and power. There are several areas of interest to the U.S. Department of Defense in this category such as batteries, capacitors, fuel cells, photovoltaics, and microturbines. Julia Phillips (SNL) discussed research needs in electronics, optoelectronics, and photonic materials. Some defense needs here can be met by using commercially developed products and processes. However, there remain a number of opportunities for further research in several areas including imaging, communications, dynamic camouflage/stealth, and health monitoring of equipment and personnel. Frank Karasz (Univ. Massachusetts) covered functional organic and hybrid materials. Karasz stressed that organic and polymeric materials will continue to make inroads in military application because of their unique combination of properties. Michael Jaffe (New Jersey Center for Biomaterials and Medical Devices) focused on materials research opportunities for bio-inspired materials, biological molecules and materials, and materials derived from biology. Schadler gave some concluding remarks and stressed that the NMAB study clearly recognizes numerous research opportunities for future defense needs. The report from the study is available online at www.nap.edu/books $/ 0309087007 / \mathrm{html}$. See also news coverage on this report in MRS Bulletin 27 (12) (December 2002), p. 947. in detail. L. Shi (Univ. Texas-Austin) presented results on thermal conductivity measurement of individual carbon nanotubes, suggesting that single-walled tubes might have thermal conductivity larger than that of diamond, currently the best thermal conductor. By contrast, as indicated by T. Borca-Tasciuc (Rensselaer Polytechnic Inst.), systems involving large ensembles of CNTs have a much lower conductivity due to a large contact resistance for the heat flow between tubes. K. Schwab (National Security Agency) described advances in fabrication and calorimetric measurement techniques allowing the observation of "quantum" effects in phonon transport with a resolution approaching that of a single quantum of thermal energy.

\section{Ion Beams Sculpt Nanostructures (See MRS Proceedings Volume 777)}

Symposium T, Nanostructuring Materials with Energetic Beams, continues a long history of MRS symposia on ion beams. In this meeting, the symposium focused on aspects of nanostructuring with energetic ion beams, including nanocrystal synthesis by ion implantation, nanostructure formation induced by radiation effects, ion irradiation through lithographic masks, ion-beam lithography, and FIBs.

The first day concentrated on ion-beam lithography and FIBs for producing various types of nanostructures. J.A. van Kan from the National University of Singapore demonstrated the lithographic abilities of $\mathrm{MeV}$ proton beams to "machine" solid materials on a scale of nanometers. Because of the long range of the proton beam, features with remarkably high aspect ratios can be obtained. Y. Liu (Univ. Virginia) discussed the use of a FIB for nanoscale patterning of PMMA resist through which extremely rapid sputter rates are achieved.

The second day of talks focused on the properties of nanoclusters formed by ionbeam patterning or ion implantation techniques. T. Devolder discussed the continuing work of the Université Paris-Sud group on nanoscale magnetic patterning, and J.E.E. Baglin from IBM's Almaden Research Center addressed the applications of ion-beam hardening to form protective shells around FePt nanoclusters. The hard, diamondlike carbon shells produced by the IBM researchers provide a rigid protective layer that prevents agglomeration of the magnetic nanoparticles. These talks were geared strongly toward applications of ion-beam nanotechnologies in the magnetic recording industry.

Symposium Support: High Voltage Engineering, Europa BV. 


\section{Smaller Can Be Stronger \\ (See MRS Proceedings Volume 778)}

Symposium U, Mechanical Properties Derived from Nanostructuring Materials, covered a broad range of materials and phenomena in the mechanical behavior of materials which have structures and features on the nanometer length scales in one, two, and three dimensions. Many presenters focused on size effects that will affect the structural alloys of the future, that is, on how macroscopic materials can be improved with nanoscale structures. These presentations showed how future alloy developments will require control of materials structure through processing, and how understanding fundamental deformation mechanisms will be required to design these new ultrahigh-strength materials (including metals, ceramics, and polymer-based composites).

Another area of interest was the deformation of small structures, where the entire material sample can be smaller than micrometers. Mechanical structures in MEMS and coatings in microelectronics were shown to be reaching a point where measuring the properties is as difficult as fabricating the structures, and many speakers demonstrated novel and exciting techniques to measure these nanometersized features, from submicron posts to nanoparticles of silicon to films deposited by atomic layer deposition.

Another area of work highlighted was the comparison between modeling deformation with either computational or analytical methods to the experimental observations and mechanistic descriptions of deformation. When the length scale becomes smaller than dislocation structures, deformation models need to grow and be adjusted to cover new and rapidly progressing observations of mechanical behavior.

Symposium Support: Hysitron and MTS Systems.

\section{Digital Learning Media Bound to SUCCEED}

The tutorial on Evaluating, Selecting, and Using Computer-Enhanced Learning Technologies and Digital Learning Materials in Engineering Education, led by J. Tront (Virginia Tech. Univ.) and B. Muramatsu (UC-Berkeley), focused on evaluating, selecting, and using digital learning media in engineering education, and was presented under the auspices of SUCCEED, an NSF-funded coalition of engineering colleges in the southeast of the United States focused on education issues for engineering. The all-day tutorial introduced attendees to a set of criteria and methods that are helpful in evaluating software and digital libraries for use in enhancing educational experiences. Noting the importance of appropriate learning techniques regardless of the media used, a portion of the session focused on good teaching practices and understanding various learning styles such as active versus reflective and visual versus verbal, and how digital media can cater to these styles.

The session was highly interactive as participants shared ideas, examined Web sites and courseware, and worked in small groups to determine areas where learning could benefit from improved digital resources. Attendees suggested several resources including software that shows the meaning of the Hall effect, databases of materials properties for plugging into problems, simple phase diagram descriptions, and processing experiences.

An interesting feature to some of the online libraries is a peer-review process for evaluating the quality of the digital resources, although there is a need for additional reviewers in the materials field (see www.merlot.org). A practical workshop was conducted in the afternoon to provide hands-on experience in applying criteria for courseware selection.

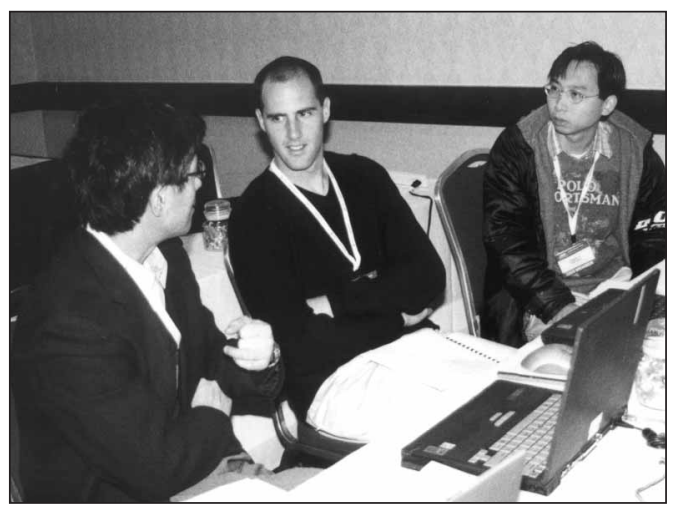

Tutorial attendees participate in SUCCEED's hands-on workshop in applying criteria for digital courseware selection.

\section{Symposium Orients Researchers in \\ Semiconductor Spintronics}

Symposium V on Semiconductor Spintronics brought together researchers on magneto-optical properties of semiconductor quantum wells and dots, singlespin control, magnetic semiconductors, electron spin transport and spin injection, nuclear polarization in semiconductors, and quantum computation.

Electrically driven electron spin resonance in a quantum well was reported using $g$-tensor modulation resonance. Instead of driving electron spins with an ac magnetic field, only a static magnetic field is used, and the $g$-tensor is modulated at $\mathrm{GHz}$ frequencies. Also described was progress in elucidating the physical processes governing spin injection from ferromagnetic metals and semiconductors, and spin transport within a nonmagnetic semiconductor.

Spin injection through a Schottky barrier was used to electrically polarize nuclei within a quantum well through dynamic nuclear polarization. High nuclear polarizations were also achieved spontaneously by optically creating carriers near a ferromagnetic/semiconductor interface. A mechanism of spontaneous nuclear polarization through spin-selective interfacial scattering was proposed and appears in agreement with experimental observations. Optical and electronic techniques for tuning nuclear coherence properties were also demonstrated, and additional ones proposed.

The materials properties of cobalt-doped titanium oxide, a high-temperature ferromagnetic semiconductor, were described. MBE growth that favors the anatase form appears most successful for generating high-quality magnetic films. Reports of high Curie temperatures for AlMnN and other zincblende materials were also presented. Theoretical considerations focused on the role of Mn interstitials and the role of disorder and spin-spin interactions in forming the magnetic ground state. Dynamic properties of magnetic semiconductors were presented, including the ability to very rapidly rotate the magnetization optically.

Theoretical proposals for quantum computation based on semiconductor spin systems included optical manipulation of single spins in quantum dots, and use of nuclear spins for ensemble quantum computation. Dramatic success in control of electronic states in quantum dots capped the symposium, including direct control of the singlet/triplet energies with lateral gates on a mesa containing two vertically coupled quantum dots.

Symposium Support: ONR/DARPA and Rockwell Scientific. 


\section{Femtosecond Pulses Prove Useful in Processing and Monitoring (See MRS Proceedings Volume 780)}

Since the inauguration of the MRS Symposium series on Advanced Optical Processing of Materials back in 1990, the number of optical-based techniques applied to process materials and the capabilities of optical systems has continued to expand and improve beyond simple PLD of thin films. Many of the most exciting areas were presented in Symposium Y. Advanced optical processing of materials now includes laser interactions with materials that are specially designed to optimize the beneficial qualities of the laser modification and, in general, femtosecondlaser processing of materials was the dominant theme.

R. Stoian (Max-Born Inst. für Nichtlineare Optik und Kurzzeitspektroskopie, Germany) showed that temporal pulse shaping of the laser pulse optimizes the femtosecond laser ablation of materials. D. Norton (Univ. Florida) gave an update on the application of PLD to a wide variety of electronic materials and H. Kim (NRL, Washington D.C.) focused on the PLD of TCOs on plastic substrates for OLEDs. M.H. Hong (Data Storage Inst., Singapore) showed how femtosecond lasers were used to create unique functional micro/nanostructures that are difficult if not impossible tasks using other conventional laser sources and manufacturing techniques. In collaboration with a research team at LZH Hannover, Germany, R. Houbertz, from the M. Popall Institute for Silicate Research, showed how real 3D submicron lithography could be performed with two-photon polymerization using inorganic-organic resins. Structures as small as $200 \mathrm{~nm}$ were generated. Combined, B.L. Davies of the Australian National University and E. Millon of Universite de Metz, France presented a comprehensive overview on the use of ultrafast and femtosecond lasers for the PLD of thin films of widely different electronic and optical materials. I. Zergioti (Institute of Electronic Structure and Laser, Greece) showed how femtosecond lasers can be used to forward transfer high-density and precise patterns of biological macromolecules including DNA, bovine serum albumin, and proteins for biosensors and multi-analyte assays.

Other novel laser-based methods and material systems were also presented. Drug delivery systems utilizing microencapsulation have been shown to dramatically improve the bio-availability and release profiles for inhaled, nasal, and oral drug delivery. J.D. Talton (Nanotherapeutics, Inc., Florida) compared the trade- offs between using conventional PLD and MAPLE for coating a fluidized bed of drug powders with biodegradable polymers, such as poly-(DL-lactide-co-glycolide), for the controlled delivery of drugs to increase their efficacy. A. Rousse (Ecole Polytechnique, France) demonstrated how the new and emerging tool of femtosecond $x$-ray diffraction allowed direct monitoring of the atomic position in a material-for example, during a phase transition-by following the change in the Bragg peak after the excitation pulse.

Symposium Support: DARPA and ARO.

\section{Evolution of Electrochemical Deposition and Corrosion Processes Studied}

Symposium Z, Mechanisms in Electrochemical Deposition and Corrosion, highlighted advances in electrodeposition of novel materials and understanding of cor-

\section{Multiscale Modeling and Experiments Continue to Uncover New Phenomena} (See MRS Proceedings Volume 779)

The topical sessions of Symposium W, Multiscale Phenomena in MaterialsExperiments and Modeling Related to Mechanical Behavior, included a diverse collection of presentations that highlighted theory, modeling, simulations, and experimental work. The symposium mainly focused on multiscale phenomena in ductile metals and alloys, and hence many of the presentations related to the individual and collective behavior of dislocations. For example, at the atomistic length scale, the work of V.V. Bulatov (LLNL, California) and co-workers offers insight into the fundamental issues associated with dislocation "glide," particularly at high velocities. As depicted in the figure, the motion of screw dislocations in bcc metals near the "Peierl's stress" may become rough, leaving in its wake large amounts of debris in the form of lattice vacancies, interstitial loops, and dislocation dipoles. The creation of this debris is spontaneous and intrinsic to the screw motion, and it agrees well with the experimental observations of dislocation self-patterning and recovery presented by P. Veyssiere (LEM,CNRS-ONERA, France) and others.

The synergistic connection between experiments and simulations was also illustrated in a session on the mechanical behavior of metallic thin films, where severe geometric constraints are known to alter dislocation behavior and govern mechanical response. In situ observations reported by T.J. Balk (Max Planck Inst. for Metals Research, Germany) and colleagues demonstrated a fundamentally different type of dislocation mechanism, "parallel glide," in ultrathin copper films. A parallel atomistic and continuum study presented by M.J. Buehler (Max Planck Inst. for Metals Research, Germany) and co-workers related the occurrence of this new mechanism to the formation of diffusion wedges by stress-assisted mass transport from the free surface to internal grain boundaries. At the mesoscopic length scale, a great deal of work is also being devoted to the development of 3D dislocation dynamics simulations that can be informed by atomistics. One example was the work of N.M. Ghoniem (UCLA) and S. Han (Princeton Univ.) that provided insight into the generation and hardening mechanisms in anisotropic multilayered thin films.

Overviews of programs at LLNL and LANL and individual contributions from various academic and national laboratories clearly demonstrated significant advances in the multiscale modeling of materials. However, this symposium also highlighted the fact that a complete understanding of the effects of composition and microstructure on the mechanical behavior of materials will only be achieved through continued highly coupled multidisciplinary work that involves both multiscale simulations and closely related

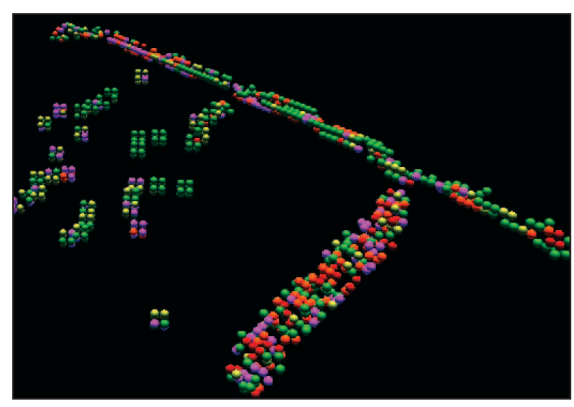

experiments. The current challenges include (1) linking of information across the length scales; and (2) successful development and coupling of experimental and simulation efforts.

Symposium Support: LLNL. 


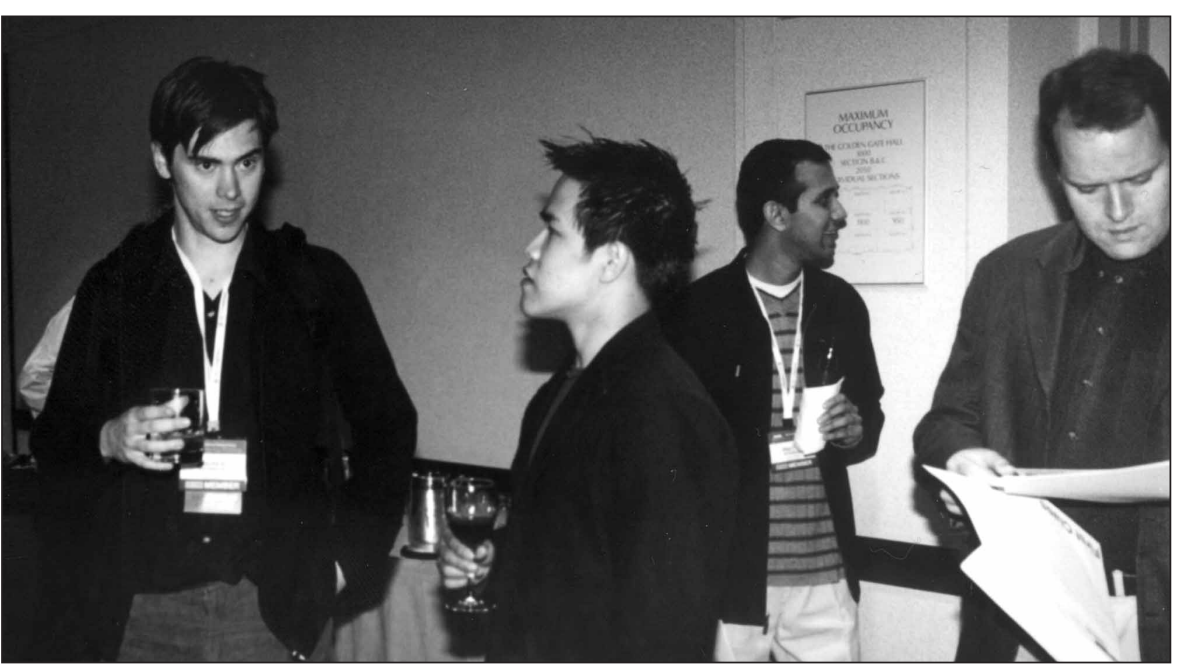

During a Student Mixer, student attendees networked with one another and other MRS members interested in student-related activities of MRS.

rosion processes. The relationship between deposition parameters, microstructure, and properties was a focus of the talks on electrochemical thin-film deposition. An emerging issue in electrodeposition is the evolution of stress during film growth, which was addressed by S. Hearne (SNL California). With the increasing use of electrodeposition for MEMS structures, the relationship between film texture and deposition conditions has become an important issue. G. Lucadamo (SNL, California) and D. Dibble (SNL, California) described the influence of additives parameters on film texture. D. Josell (NIST, Maryland) described a model for superfilling in the deposition of copper metallization based on curvature enhanced coverage of the accelerator. Electrodeposition of metal oxides was discussed by K. Stevenson (UT-Austin) and R. Diaz (Univ. Barcelona, Spain). E. Walter (UCIrvine) received a graduate student gold award from MRS for his work on electrodeposition of beaded nanowires. J. Guyer (NIST, Maryland) presented preliminary work on the phase-field modeling of electrochemical interfaces, illustrating that electrocapillary curves and capacitance can be obtained using this approach. K. Sieradzki (ASU) described the role of selective etching or dealloying on the corrosion of alloys. 